\title{
A flexible three-dimensional stratocumulus, cumulus and cirrus cloud generator (3DCLOUD) based on drastically simplified atmospheric equations and the Fourier transform framework
}

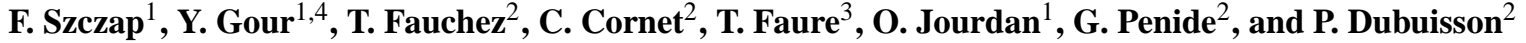 \\ ${ }^{1}$ Laboratoire de Météorologie Physique, UMR6016, CNRS, Aubière, France \\ ${ }^{2}$ Laboratoire d'Optique Atmosphérique, UMR8518, CNRS, Villeneuve d'Ascq, France \\ ${ }^{3}$ Laboratoire d'Ingénierie pour les Systèmes Complexes, Clermont-Ferrand, France \\ ${ }^{4}$ Laboratoire de Météorologie Physique, Institut Universitaire de Technologie d'Allier, Montluçon, France
}

Correspondence to: F. Szczap (szczap@opgc.univ-bpclermont.fr)

Received: 31 October 2013 - Published in Geosci. Model Dev. Discuss.: 14 January 2014

Revised: 4 June 2014 - Accepted: 25 June 2014 - Published: 25 August 2014

\begin{abstract}
The 3DCLOUD algorithm for generating stochastic three-dimensional (3-D) cloud fields is described in this paper. The generated outputs are 3-D optical depth $(\tau)$ for stratocumulus and cumulus fields and 3-D ice water content (IWC) for cirrus clouds. This model is designed to generate cloud fields that share some statistical properties observed in real clouds such as the inhomogeneity parameter $\rho$ (standard deviation normalized by the mean of the studied quantity), the Fourier spectral slope $\beta$ close to $-5 / 3$ between the smallest scale of the simulation to the outer $L_{\text {out }}$ (where the spectrum becomes flat). Firstly, 3DCLOUD assimilates meteorological profiles (humidity, pressure, temperature and wind velocity). The cloud coverage $C$, defined by the user, can also be assimilated, but only for stratocumulus and cumulus regime. 3DCLOUD solves drastically simplified basic atmospheric equations, in order to simulate 3-D cloud structures of liquid or ice water content. Secondly, the Fourier filtering method is used to constrain the intensity of $\rho, \beta, L_{\text {out }}$ and the mean of $\tau$ or IWC of these 3-D cloud structures. The 3DCLOUD model was developed to run on a personal computer under Matlab environment with the Matlab statistics toolbox. It is used to study 3-D interactions between cloudy atmosphere and radiation.
\end{abstract}

\section{Introduction}

Clouds have a significant effect on the Earth radiation budget. They reflect the solar radiation and reduce the warming of the Earth (albedo effect). They also create a greenhouse effect by trapping the thermal radiation emitted from the Earth's surface, reducing the radiative cooling of the Earth (Collins and Satoh, 2009). Cloud feedback has remained, however, the largest uncertainty in the study of climate sensitivity for almost 20 years (Bony et al., 2006). In almost all climate models, clouds are assumed plane- parallel with homogeneous optical properties (PPH), and radiation codes use a one-dimensional (1-D) scheme. Therefore, improving parameterisations of clouds in large-scale model, especially their interaction with radiation, is a challenge in order to reduce uncertainty in model projections of the future climate (Illingworth and Bony, 2009). Improved global characterization of the three dimensional (3-D) spatial distribution of clouds is, thus, necessary (Clothiaux et al., 2004).

Moreover, satellite passive sensors, such as multi-spectral and multi-angular radiometers, and satellite active sensor, such as LIDAR and RADAR in the A-train mission, allow the retrieval of cloud horizontal and vertical optical properties with an adequate spatial and temporal coverage. For practical and computational cost purposes, interpretation of such measurements generally also assumes 1-D radiative algorithm and PPH cloud. This assumption can be far from being realistic and leads to biases on the retrieved properties from passive sensors (Barker and Liu, 1995; Várnai and 
Marshak, 2002, 2007; Lafont and Guillemet, 2004; Cornet et al., 2005, 2013) and active sensors (Battaglia and Tanelli, 2011). These biases depend at least, on the cloud coverage and on the variability of cloud optical depth or water content. This variability is quantified by an inhomogeneity parameter, often defined as the standard deviation normalized by the mean of the studied quantity (Szczap et al., 2000; Carlin et al., 2002; Oreopoulos and Cahalan, 2005; Sassen et al., 2007; Hill et al., 2012).

Determining the significance of the 3-D inhomogeneity of clouds for climate and remote sensing applications requires the measurement and the simulation of the full range of actual cloud structure. Apart from the computational time, accurate 3-D cloudy radiative transfer problem is not an issue, per se (Evans and Wiscombe, 2004). Monte Carlo transfer models can indeed accurately and efficiently compute radiative properties for arbitrary cloud fields (Battaglia and Mantovani, 2005; Pincus and Evans, 2009; Mayer, 2009; Cornet et al., 2010, 2013; Battaglia and Tanelli, 2011; Fauchez et al., 2013). The difficulty is to generate cloud property fields that are statistically representative of cloud fields in nature.

Cloud fields generated by dynamic cloud models, such as the cloud resolving model (CRM) or the large-eddy simulation model (LES), are very attractive, as they contain the state of the art of physical processes (resolution of atmospheric equations, detailed microphysics, radiation, etc.). The goal of the LES approach is to simulate the three-dimensional atmospheric turbulent flows. There are different scales of turbulent eddies; large eddies (from 100 to $1000 \mathrm{~m}$ and more) that are produced directly by the instability of the mean flow and small eddies (from a few centimetres to $100 \mathrm{~m}$ ) as well as by the energy-cascade process from the larger eddies (Moeng, 1984). LES seeks to capture accurately the larger eddies, while only modelling the smaller ones. Instead of reproducing all the scales of turbulence flow, they can integrate a flow in which small scale details are removed from the solution. The spatial filtered equations can, therefore, be integrated with available resources (Bryan et al., 2003). Nevertheless, they are still very expensive to run in a 3-D domain.

Stochastic models have the capability to simulate quickly realistic 2-D and 3-D cloud structures with just a few parameters. Examples of these types of cloud models are: the bounded cascade model (Cahalan et al., 1994; Marshak et al., 1998), the iterative amplitude adapted Fourier transform (IAAFT) algorithm (Venema et al., 2006), the SITCOM model (Di Guiseppe and Thompkins, 2003), the tree-driven mass accumulation process (tdMAP) model (Benassi et al., 2004), the model developed by Evans and Wiscombe (2004) for low liquid clouds (stratocumulus and cumulus) or by Alexandrov et al. (2010) and the Cloudgen model (Hogan and Kew, 2005) for high ice clouds (cirrus). These stochastic models are based on fractal or Fourier framework. The scale invariant properties observed in real clouds can be controlled. The power spectra of the logarithm of their optical properties (optical depth, liquid water content or liquid water path for low clouds and ice water content for high clouds) typically exhibits a spectral slope of around -5/3 (Davis et al., 1994, 1996, 1997, 1999; Cahalan et al., 1994; Benassi et al., 2004; Hogan and Kew, 2005; Hill et al., 2012; Fauchez et al., 2014) from small scale (a few metres) to the "integral scale" or the outer scale (few tenths of a kilometre to one-hundred kilometres), where the spectrum becomes flat (i.e. decorrelation occurs). The disadvantage of such models arises from the fact that effects of meteorological processes are not always considered and dominant scales of organization related to turbulent eddy due, for example, to wind shear, convection, and entrainment are not directly modelled. At the same time, it should be noted that Cloudgen does consider the effect of wind shear on cirrus cloud.

The aim of the 3DCLOUD algorithm is to reconcile these two approaches. In Sect. 2, we describe the 3DCLOUD generator. In Sect. 3, 3DCLOUD outputs are compared to LES outputs to check the validity of the chosen basic atmospheric equations. In Sect. 4, stratocumulus, cumulus and cirrus examples provided by 3DCLOUD are presented.

\section{The 3DCloud generator}

3DCLOUD generates, in two distinct steps (see Fig. 1), a 3-D optical depth field for stratocumulus and cumulus or a 3-D ice water content field for cirrus clouds. These cloud fields were chosen as most of the papers dealing with scale invariant properties focus on liquid water path and optical depth for stratocumulus and cumulus and on ice water content for cirrus. During the first step, meteorological vertical profiles (temperature, pressure, wind, humidity), defined by the user, are assimilated and basic atmospheric equations are resolved. During the second step, cloud scale invariant properties are constrained in a Fourier framework. At the same time, a gamma distribution of local optical depth or 3-D ice water content (IWC) is mapped onto the liquid water content (LWC), or IWC, generated during the first step. This gamma distribution is iteratively computed in such a way that the mean optical depth or IWC and the inhomogeneity parameter satisfy the values imposed by the user. Details of these two steps are presented below.

\subsection{Step 1: the 3-D LWC/IWC generator}

The essential basic quantities to generate cloud fields are the condensed water mixing ratio $q_{\mathrm{c}}=q_{1}+q_{\mathrm{i}}$ where $q_{\mathrm{l}}$ is the liquid water mixing ratio and $q_{\mathrm{i}}$ is the ice water mixing ratio, the wind velocity vector $\boldsymbol{u}$, air pressure $p$, temperature $T$, and vapour water mixing ratio $q_{\mathrm{v}}$. Mixing ratios are the mass of vapour or condensed water per unit of dry air mass. We describe in this section the equations used to generate clouds with the associated simplifications used. 


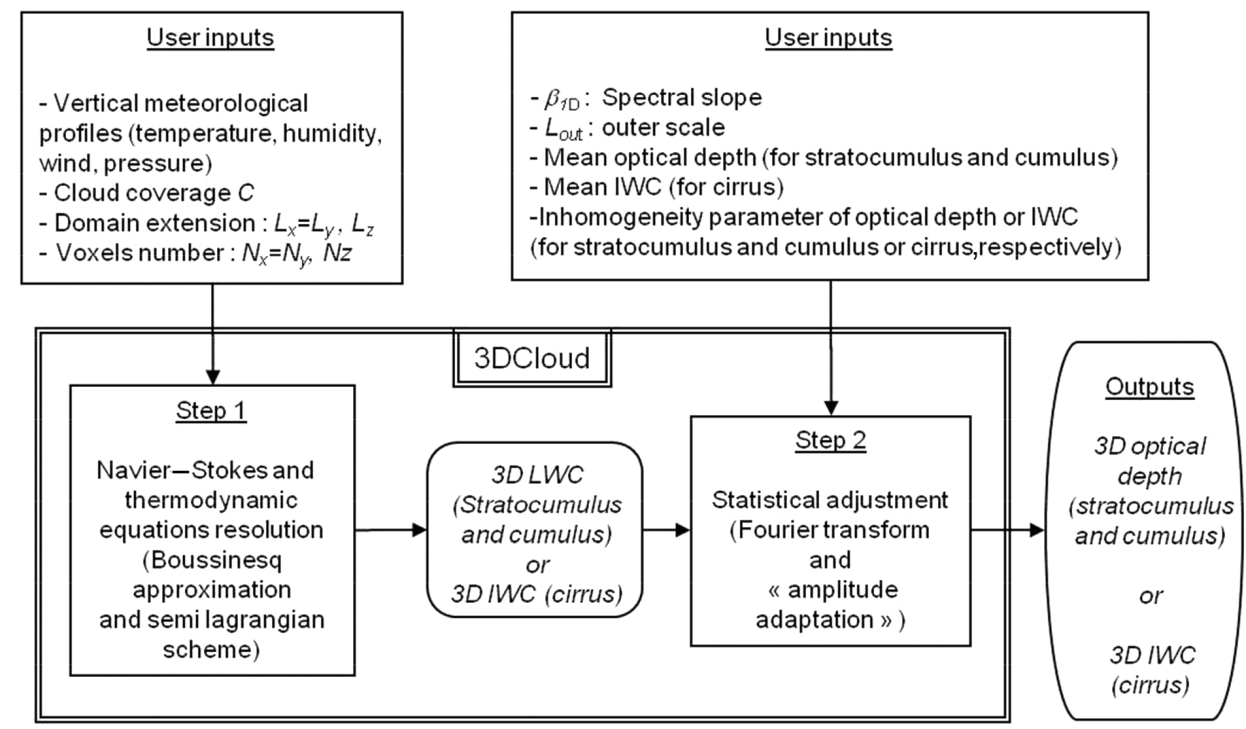

Figure 1. General flow chart of the stratocumulus, cumulus and cirrus generator 3DCloud. Note that 3DCloud algorithm is divided in two distinct steps.

\subsubsection{The simplification of basic atmospheric equations}

The continuity and momentum equations of the atmosphere can be written as follows (Houze, 1993):

$\left\{\begin{array}{l}\frac{\mathrm{d} \rho}{\mathrm{d} t}=-\rho \nabla \cdot \boldsymbol{u} \\ \frac{\mathrm{d} \boldsymbol{u}}{\mathrm{d} t}=-\frac{1}{\rho} \nabla p-f \boldsymbol{k} \wedge \boldsymbol{u}-g \boldsymbol{k}+\boldsymbol{F},\end{array}\right.$

where $t$ is time, $\rho$ the air density, $f$ the Coriolis parameter, $g$ the acceleration due to gravity and $\boldsymbol{F}$ the acceleration due to other forces (frictional acceleration for example). $D / \mathrm{d} t=\partial / \partial t+\boldsymbol{u} \cdot \nabla$ is the Lagrangian derivative operator following a parcel of air, $\partial / \partial t$ is the Eulerian derivative operator and $\nabla$ is the three-dimensional gradient operator. $\boldsymbol{u}=u \boldsymbol{i}+v \boldsymbol{j}+w \boldsymbol{k}$ is the wind velocity vector with horizontal components $u, v$ and vertical component $w$ projected in the Cartesian geometry system, where $\boldsymbol{i}, \boldsymbol{j}$ and $\boldsymbol{k}$ are the unit vectors in the $x, y$ and $z$ directions. The continuity and momentum equations of the atmosphere under the anelastic and Boussinesq approximation, assuming shallow motion, neglecting Coriolis parameter, neglecting frictional forces, and neglecting the molecular viscosity can be written as follows (Holton, 2004, p. 117; Houze, 1993, p. 35; Emanuel, 1994, p. 11):

$\left\{\begin{array}{l}\nabla \cdot \boldsymbol{u}=0 \\ \frac{\mathrm{d} \boldsymbol{u}}{\mathrm{d} t}=-\frac{1}{\rho_{0}} \nabla p^{*}+B \boldsymbol{k},\end{array}\right.$

where $B$ is the buoyancy acceleration, $\rho_{0}$ is the constant mean value of air density and $p^{*}$ is the pressure perturbations. The above differential operators are valid only in the limit when $\delta t, \delta x, \delta y$ and $\delta z$ approach 0 (Pielke, 2002, p. 41). Nevertheless, turbulent motions (shear induced eddies, convection eddies) have spatial and temporal variations at scales much smaller than those resolved by LES and 3DCLOUD. If we assume field variables can be separated in slowly varying mean field and rapidly varying turbulent component, and if we apply the Reynolds decomposition, we can rewrite the above equation set as

$\left\{\begin{array}{l}\nabla \cdot \boldsymbol{u}=0 \\ \frac{\mathrm{d} \boldsymbol{u}}{\mathrm{d} t}=-\frac{1}{\rho_{0}} \nabla p^{*}+B \boldsymbol{k}+\boldsymbol{\Phi},\end{array}\right.$

where $\boldsymbol{\Phi}$ is the three dimensional convergence of the eddy flux of moment (Houze, 1993, p. 42), the turbulent flux (Holton, 2002, p. 119) or the sub-grid correlation term (Pielke, 2002, p. 44). The Reynolds decomposition is not used in LES. The atmospheric equations are derived by spatial filtering, where a special function is applied. Thus, the filtering operation acts on atmospheric quantities and separates them in two categories: the resolved one (large eddy) and unresolved one (subgrid scale). An unknown term remains in the filtered equations of LES, often called the subgrid-scale stress, which needs to be parameterized or estimated with the help of subgrid-scale modelling. This subgrid-scale stress for LES equations is analogous to the $\boldsymbol{\Phi}$ term for Reynolds decomposition. In 3DCLOUD, the $\boldsymbol{\Phi}$ term is voluntarily neglected. Indeed, the guiding idea of 3DCLOUD is to simulate, in the fastest way, 3-D fluctuations of LWC/IWC of a cloud showing turbulent properties (or invariant scale properties).

When water phase changes are only associated with condensation and evaporation (or sublimation), the first law of thermodynamics can be written (Houze, 1993) as follows:

$\frac{\mathrm{d} \theta}{\mathrm{d} t}=-\frac{L}{C_{p} \prod} \frac{\mathrm{d} q_{\mathrm{v}}}{\mathrm{d} t}$, 
where $L=2500 \mathrm{~kJ} \mathrm{~kg}^{-1}$ and $L=2800 \mathrm{~kJ} \mathrm{~kg}^{-1}$ are the usual latent heat of vaporization of water and ice, respectively. $C_{p}=1.004 \mathrm{~kJ} \mathrm{~kg}^{-1} \mathrm{~K}^{-1}$ is the usual specific heat of dry air at constant pressure, $\theta$ is the potential temperature and $\prod=$ $\left(p / p_{0}\right)^{\kappa}=T / \theta$ is the Exner function where $p_{0}=1000 \mathrm{hPa}$ and $\kappa=0.286$. In addition to the equation of motion and the first law of thermodynamics, air parcels follow the water continuity equation:

$$
\frac{\mathrm{d} q_{\mathrm{i}}}{\mathrm{d} t}=S_{i}, i=1, \ldots, n,
$$

where $S_{i}$ are the sum of the sources and sinks for a particular category (among $n$ categories) of water indicated by $i$ (vapour, solid, liquid water category for example).

As the horizontal extension of the simulated cloud fields is around a few $\mathrm{km}$, horizontal pressure is assumed to be constant. Therefore, the current version of 3DCLOUD does not have a large enough domain to contain power in the mesoscale. All these considerations lead to a dramatic simplification of the dynamic equations. The simplified equations of 3DCLOUD governing the formation of 3-D cloud structures are

$$
\left\{\begin{array}{l}
\frac{\mathrm{d} \boldsymbol{u}}{\mathrm{d} t}=g\left(\frac{\theta_{\mathrm{v}}^{*}}{\theta_{v 0}}-q_{\mathrm{c}}\right) \boldsymbol{k}-\frac{\mathbf{1}}{\rho_{\mathbf{0}}} \frac{\partial p^{*}}{\partial z} \boldsymbol{k} \\
\nabla \cdot \boldsymbol{u}=0 \\
\frac{\mathrm{d} \theta}{\mathrm{d} t}=\frac{L}{C_{p} \Pi} \\
\frac{\mathrm{d} q_{\mathrm{v}}}{\mathrm{d} t}=-\xi \\
\frac{\mathrm{d} q_{\mathrm{c}}}{\mathrm{d} t}=\xi
\end{array}\right.
$$

where the reference state is denoted by subscript 0 and the deviation from the reference state by an asterisk, $\theta_{\mathrm{v}}=$ $\theta\left(1+0.61 q_{\mathrm{v}}\right)$ is the virtual potential temperature. For stratocumulus and cumulus fields $\xi$ is estimated as follows:

$\xi=\min \left(q_{\mathrm{vs}}-q_{\mathrm{v}}, q_{\mathrm{c}}\right) \Delta t(7)$, where $\Delta t$ is the simulation time step and $q_{\mathrm{vs}}(T, p)$ is the saturation mixing ratio derived from Thetens and Magnus formula: $q_{\mathrm{vs}}(T, p)=$ $\frac{0.622 P_{\text {sat }}}{\left(p / 100-0.378 P_{\text {sat }}\right)}$, where $P_{\text {sat }}=6.107 \exp \left[\frac{4028(T-273.15)}{234.82(T-38.33)}\right]$ for water, $P_{\text {sat }}=6.107 \times 10^{\left[\frac{9.5(T-273.15)}{265.5+(T-273.15)}\right]}$ for ice. Computation of $\xi$ at each simulation step is based on the work of Asai (1965). For cirrus clouds, condensation, evaporation and ice crystals sedimentation processes are very complex and still not well understood (Kärcher and Spichtinger, 2009). In order to take into account super-saturation and subsaturation regions in cirrus clouds in a very simple way, we used the parameterisation of Starr and Cox (1985) to compute the values of $\xi$ every $2.5 \mathrm{~min}$. Sedimentation processes are taken into account in Eq. (5.5) by adding ice fall speed $v_{\text {fall }}$ taken from Starr and Cox (1985):

$v_{\text {fall }}=\frac{1.5}{6} \log 10\left[\max \left(\mathrm{IWC}, 1 \times 10^{-6}\right)\right]+1.5$,

where $v_{\text {fall }}$ is in $\mathrm{ms}^{-1}$ and the ice water content IWC in $\mathrm{g} \mathrm{m}^{-3}$.

\subsubsection{Assimilation of meteorological profiles and cloud coverage}

In order to control the structure and nature of clouds and especially vertical position and extension, it is necessary to impose a large-scale environment. Practically, forcing terms are added to the 3DCLOUD equations to nudge the solutions towards observations. Our state observations are the initial meteorological profiles (provided by the user for example) and do not change during the simulation. The technique used is based on the initialization integration method (Pielke, 2002). Consequently, 3DCLOUD equations become

$$
\left\{\begin{array}{l}
\frac{\mathrm{d} u}{\mathrm{~d} t}=G_{u}(z)\left[u_{\mathrm{ini}}(z)-\bar{u}(z)\right] \\
\frac{\mathrm{d} v}{\mathrm{~d} t}=G_{\mathrm{v}}(z)\left[v_{\mathrm{ini}}(z)-\bar{v}(z)\right] \\
\frac{\mathrm{d} w}{\mathrm{~d} t}=g\left(\frac{\theta_{\mathrm{v}}^{*}}{\theta_{v 0}}-q_{\mathrm{c}}\right)-\frac{1}{\rho_{0}} \frac{\partial p^{*}}{\partial z} \\
\nabla \cdot \boldsymbol{u}=0 \\
\frac{\mathrm{d} \theta}{\mathrm{d} t}=\frac{L}{C_{p} \Pi} \xi+G_{\theta}(z)\left[\theta_{\mathrm{ini}}(z)-\theta\right] \\
\frac{\mathrm{d} q_{\mathrm{v}}}{\mathrm{d} t}=-\xi+G_{q_{\mathrm{v}}}(z)\left[q_{v_{\text {ini }}}(z)-q_{\mathrm{v}}\right] \\
\frac{\mathrm{d} q_{\mathrm{c}}}{\mathrm{d} t}=\xi
\end{array}\right.
$$

where for variables $X, \bar{X}(z)$ is the mean of $X$ at height $z$ and quantities $G_{X}(z)$ are adjusted during the simulation in such a way that $X$ or $\bar{X}(z)$ do not diverge far from the initial conditions $X_{\text {ini }}(z)$. In a general way, $G$ is the inverse of timescale but, because the contribution of $G$ is artificial, it must not be a dominant term in the governing equations and should be scaled by the slowest physical adjustment processes in the model (Cheng et al., 2001). This timescale was first set to $1 \mathrm{~h}$ but this value was found to be too large and must be adjusted as a function of altitude, especially at height where large vertical gradients of $X$ appear (e.g. at the top of a stratocumulus cloud, for example). Therefore, we developed a very fast and simple numerical method to adjust the values of $G_{X}(z)$ during the simulation. At each level, we compute the relative difference $\alpha_{X}(z)=\frac{X_{\text {ini }}(z)-\bar{X}(z)}{\bar{X}(z)}$. 100. $G_{X}(z)$ is assumed to be proportional to $\alpha_{X}(z)$ and is estimated as $G_{X}(z)=\min \left[G_{\min }+\frac{G_{\max }-G_{\min }}{\alpha_{X, \max }} \alpha_{X}(z), G_{\max }\right]$ where $G_{\min }=\frac{1}{3600} \mathrm{~s}^{-1}$ and $G_{\max }=\frac{1}{2 \Delta t}$. Values of $\alpha_{X, \max }$ were estimated during our numerous numerical experiments. For stratocumulus and cumulus, $\alpha_{X, \max }$ values for horizontal wind, temperature and humidity are $20 \%, 2 \%$ and $20 \%$ respectively and for cirrus, $\alpha_{X, \max }$ values are $20 \%, 10 \%$ and $10 \%$, respectively.

The cloud coverage $C$ is defined as the fraction of the number of cloudy pixels to the total number of pixels in the 2-D horizontal plan. The value of $C$ is chosen with the assimilation of initial meteorological profiles. At each time step, the initial profile of vapour mixing ratio $q_{v_{\text {ini }}}(z)$ is modified between cloud base and top height if $C \geq 50 \%$ or between ground and cloud top height if $C<50 \%$ until $C$ agrees with the desired value within few percents. The new "initial" profile of vapour mixing ratio $q_{v_{\text {ini }}}^{\text {new }}(z)$ is computed from 
the currently simulated (old) profile of vapour mixing ratio $q_{v_{\text {ini }}}^{\text {old }}(z)$ as $q_{v_{\text {ini }}}^{\text {new }}(z)=q_{v_{\text {ini }}}^{\text {old }}(z) \pm \frac{n_{z}-n_{\text {base }}}{n_{\text {top }}-n_{\text {base }}} q_{v_{\text {ini }}}^{\text {old }}(z) \times \frac{0.1}{100}$ where $z$ is height, and $n_{z}, n_{\text {top }}$ and $n_{\text {base }}$ are the levels indexes (in $z$ direction) corresponding to cloud top height and to cloud base height (or ground), respectively.

This method gives satisfactory results for stratocumulus and cumulus cloud fields (see Sect. 4.1.2), but not for cirrus fields. This is because condensation/evaporation and dynamic processes are different for stratocumulus/cumulus and cirrus regimes. Indeed, for liquid and warm stratocumulus/cumulus regime, liquid super or sub-saturation regions are not allowed in 3DCLOUD. Therefore, the distinction between cloudy and free cloud voxels is sharp. Moreover, as stratocumulus/cumulus fields are often driven by convection processes in a well-mixed planetary boundary layer, vertical correlation occurs between cloudy voxels (free cloud voxels) and updrafts (downdraughts). Thus, the fractional cloud coverage is easily controlled by adjusting the vertical profile of vapour mixing ratio during the simulation. By contrast, in ice cirrus regimes, (large) ice crystals can survive even if ice relative humidity is less than $100 \%$. Ice super or subsaturation regions are often observed in cirrus and are taken into account in the Starr and Cox parameterization used in 3DCLOUD. Therefore, many cloudy voxels still exist in our cirrus simulations, even if the ice water content is very small. The distinction between cloudy and free cloud voxels is, thus, very tenuous. Moreover, cirrus dynamics are often driven by wind shear; small fractional cloud coverage can exist at the top of the cirrus field due to convection or radiative cooling coexisting with large fractional cloud coverage and can also exist at the bottom of cirrus field due to wind shear. Finally, the total cloud coverage could be large. If we adjust the vertical profile of vapour mixing ratio during the simulation in the same way as for the stratocumulus/cumulus field, the total cloud coverage will be difficult to control. Further investigations are thus needed to perfectly control the cloud coverage of cirrus simulated by 3DCLOUD.

\subsubsection{Implementation of 3DCLOUD algorithm}

To implement the previously described equations, space is divided in $\left(N_{x}+2\right) \times\left(N_{y}+2\right) \times\left(N_{z}+2\right)$ cells or voxels where $N_{x}, N_{y}$ and $N_{z}$ are the voxel numbers in each direction. A voxel is characterized by its spatial resolution with $\Delta x=\Delta y \neq \Delta z$. Horizontal extensions are $L_{x}=L_{y}$ and can be different from the vertical extension $L_{z}$. In order to take into account the boundary conditions, one layer of voxel is added around the simulation domain.

A semi-Lagrangian scheme was chosen to solve the equation:

$\frac{\mathrm{d} X}{\mathrm{~d} t}=\frac{\partial X}{\partial t}+\boldsymbol{u} \cdot \nabla X=0$

where $X$ is a scalar advected by the wind velocity $\boldsymbol{u}$. $X$ can be the potential temperature $\theta$, the condensed water $q_{\mathrm{c}}$ or the vapour mixing ratios $q_{\mathrm{v}}$, and also the three components of wind velocity $u, v$ and $w$. Two steps are needed in order to compute the value of $X(x, t+\Delta t)$ at a fixed position $x$ and at time $t+\Delta t . X(x, t)$ and $\boldsymbol{u}(x, t)$ are known values and $\Delta t$ is the time step. First, we compute the previous position $p(X, t-\Delta t)=x-\boldsymbol{u}(x, t) \Delta t$ of $X$ at time $t-\Delta t$. In a second step, we compute the value of $X(p, t)$ at the position $p$ and at the time $t$ by an interpolation scheme. This interpolated value $X(p, t)$ is the desired value $X(x, t+\Delta t)$. The main advantage of this approach is that the time step is not restricted by the Courant-Friedrichs-Lewy stability limit, but by the less restrictive condition that parcels do not overtake each other during the time step (Riddaway, 2001). Therefore, at each iteration, the maximum value of time step $\Delta t_{\max }$ can be roughly estimated as $\Delta t_{\max }=$ $1 /(\max |\Delta u / \Delta x|+\max |\Delta v / \Delta y|+\max |\Delta w / \Delta z|)$. The accuracy of this approach depends on the accuracy of the interpolation scheme. Due to CPU time, we chose a linear interpolation, which, unfortunately, provides numerical dissipations. However, this drawback is overcome using the Fourier transform performed in the second step of the 3DCLOUD algorithm (see Sect. 2.2.2).

As the Fourier transform is easy to implement, this method was chosen to solve the equation $\nabla \cdot \boldsymbol{u}=0$. In the Fourier domain, the gradient operator $\nabla$ is equivalent to the multiplication by $i \boldsymbol{k}$, where $i \equiv \sqrt{-1}$ and $\boldsymbol{k}$ is the wave number vector. Thus, the following equation $i \boldsymbol{k} \cdot \hat{\boldsymbol{u}}(\boldsymbol{k})=0$, where $\hat{\boldsymbol{u}}$ is the transform of wind velocity $\boldsymbol{u}$ in the Fourier domain, has to be solved. This implies that the Fourier transform of the velocity of a divergent free field is always perpendicular to its wave numbers. Therefore, the quantity $1 / \boldsymbol{k}^{2}(\boldsymbol{k} \cdot \hat{\boldsymbol{u}}(\boldsymbol{k})) \boldsymbol{k}$ is removed from $\hat{\boldsymbol{u}}$. Keeping the real part of inverse transform of $\hat{\boldsymbol{u}}$ provides the new wind velocity $\boldsymbol{u}$ with the desired free divergent property.

Lateral periodic conditions and continuity conditions to bottom and top are applied. For wind velocity, free slip boundary conditions are applied at the bottom and top of the domain, which are assumed to be a solid wall (i.e. $w=0$ ). But, as the Fourier transform (which is needed to solve the equation $\nabla \cdot \boldsymbol{u}=0$ ) requires periodic conditions, it provides spurious oscillations during the simulations. In order to limit this effect, extra levels with wind velocity set to zero are added under and above the model domain.

The 3DCLOUD algorithm to simulate 3-D structures of liquid water content (LWC) or IWC is, in summary:

1. Definition of initial meteorological profiles $u_{\text {ini }}, v_{\text {ini }}$, $\theta_{\text {ini }}, q_{v_{\text {ini }}}$ from idealized cloud conceptual models or from the user. The vertical pressure profile is generally computed from the hydrostatic law, but can be provided by the user.

2. Initial perturbations $\boldsymbol{u}^{\prime}$ are added to wind velocity $\boldsymbol{u} . \boldsymbol{u}^{\prime}$ is free-divergent and turbulent with a spectral slope of $-5 / 3$ (see more explanations in Sect. 2.2.2). 
3. Assimilation of initial meteorological profiles (optional, see Eq. 9 and Sect. 2.1.2).

4. Constrain of divergent-free velocity $\boldsymbol{u}$ (see Eq. 9).

5. Computation of ice fall speed $v_{\text {fall }}$ (only for cirrus cloud, see Eq. 8).

6. Advection of $u, v, w, \theta, q_{\mathrm{v}}$ and $q_{\mathrm{c}}$ by wind velocity $\boldsymbol{u}$ (see Eq. 10).

7. Modification of $\theta, q_{\mathrm{v}}$ and $q_{\mathrm{c}}$ due to evaporation/condensation processes (see Eq. 7).

8. Modification of the vertical velocity due to buoyancy (see Eq. 9).

9. Modification of $q_{v_{\text {ini }}}$ in order to assimilate cloud coverage $C$ (optional, only for stratocumulus and cumulus, see Sect. 2.1.2).

10. Return to (3) until maximum iteration number is reached.

\section{Computation of LWC or IWC.}

\subsection{Step 2: statistical adjustment}

Hereafter, we present the second step of the 3DCLOUD algorithm that is the methodology to adjust, according to user requirements, the mean optical depth $\bar{\tau}$ (or the mean ice water content $\overline{\mathrm{IWC}}$ ) and the inhomogeneity parameter of the optical depth $\rho_{\tau}$ (or the ice water content $\rho_{\text {IWC }}$ ) from the LWC (or from the IWC) simulated at the step 1. The distribution of $\tau$ or IWC is assumed to follow a gamma distribution. Indeed, distribution of $\tau$ and IWC are usually well represented by a lognormal or gamma distribution (Cahalan et al., 1994; Barker et al., 1996; Carlin et al., 2002; Hogan and Illingworth, 2003; Hogan and Kew, 2005). The scale invariant cloud properties, controlled at each level, are characterised by the spectral exponent $\beta_{1-\mathrm{D}}$ close to $-5 / 3$ (slope $\beta$ of the one dimension wave number spectrum in $\log -\log$ axes of the Fourier space). This spectral slope is computed from the outer scale $L_{\text {out }}$ (defined by the user) to the smaller scale (voxel horizontal size).

\subsubsection{Control of the mean and of the inhomogeneity parameter}

The relation between local optical depth $\tau(x, y, z)$, liquid water path (LWP) and density of water $\rho_{1}$ in each voxel is given by (Liou, 2002)

$$
\begin{aligned}
\tau(x, y, z)= & \frac{3}{2 \rho_{1}} \frac{\operatorname{LWP}(x, y, z)}{R_{\text {eff }}} \text { with } \operatorname{LWP}(x, y, z)= \\
& \rho_{\text {air }} q_{\mathrm{c}}(x, y, z) \Delta z,
\end{aligned}
$$

where $x, y, z$ are the spatial positions inside the simulation domain, $\operatorname{LWP}(x, y, z)$ is the local liquid water path and $\Delta z$ is the vertical resolution. Local quantity means that the quantity is estimated inside a voxel. The optical depth $\tau(x, y)$ for each pixel is the sum of local optical depths along the $z$ axis:

$\tau(x, y)=\sum_{z=1}^{N_{z}}(x, y, z)$.

The mean optical depth $\bar{\tau}$ is then defined as follows:

$\bar{\tau}=\frac{1}{N_{x} N_{y}} \sum_{x=1}^{N_{x}} \sum_{y=1}^{N_{y}} \tau(x, y)$.

In the same way, for ice cloud, the mean IWC is obtained with

$\overline{\mathrm{IWC}}=\frac{1}{N_{x} N_{y} N_{z}^{*}} \sum_{x=1}^{N_{x}} \sum_{y=1}^{N_{y}} \sum_{z=1}^{N_{z}^{*}} \operatorname{IWC}(x, y, z)$,

where $N_{z}^{*}$ is the number of layers between cloud top and cloud bottom.

To describe the amplitude of the optical depth for 1-D and 2-D overcast cloud, Szczap et al. (2000) defined the inhomogeneity parameter of optical depth $\rho_{\tau}$. For 3-D broken fields, this parameter is defined according to

$\rho_{\tau}=\frac{\sigma\left[\tau^{>0}(x, y)\right]}{\bar{\tau}^{>0}(x, y)}$,

where $\sigma\left[\tau^{>0}(x, y)\right]$ and $\bar{\tau}^{>0}(x, y)$ are the standard deviation and the mean of the strictly positive optical depth.

Due to the flexibility of the mathematical formulation of the gamma distribution and to its ability to mimic the attributes of other positive-value distributions, such as lognormal and exponential distributions, we choose to control $\bar{\tau}$ or $\overline{\text { IWC }}$ and $\rho_{\tau}$ or $\rho_{\text {IWC }}$ by mapping theoretical gammadistributed properties onto the simulated properties. This mapping technique is analog to the "amplitude adaptation" technique explained in Venema et al. (2006), where amplitudes are adjusted based on their ranking. The gamma distribution is a two-parameter family of continuous probability distribution. It has a shape parameter $a$ and scale parameter $b$. The equation defining the probability density as a function of a gamma-distributed random variable $Y$ is as follows:

$Y=f(\mu ; a, b)=\frac{1}{b^{a} \Gamma(a)} \mu^{a-1} e^{-\mu / b}$,

where $\Gamma$ (.) is the gamma function. We develop a simple iterative algorithm, where values of $a$ and $b$ are adjusted until mean and inhomogeneity parameters reach the required user values within few percents.

\subsubsection{Control of invariant scale properties by adjustment of spectral exponent in Fourier space}

The spectral slope value $\beta_{1-\mathrm{D}}$ of the horizontally 2-D field is adjusted according to the following methodology. As proposed by Hogan and Kew (2005), we choose to manipulate 
the 2-D plan of Fourier amplitudes of local optical depth $\tau_{2-\mathrm{D}}\left(\right.$ or $\left.\mathrm{IWC}_{2-\mathrm{D}}\right)$ with a 2-D Fourier transform performed at each height of cloudy layer. Suppose a 2-D isotropic field $g(x, y)$ characterized by a Gaussian probability density function (PDF) and a 1-D power spectrum $E_{1}(k)$ with a spectral slope $\beta_{1-\mathrm{D}}$ at all scales defined as follows:

$E_{1}(k)=\widehat{E_{1}} k^{-\beta_{1-\mathrm{D}}}$,

where $k$ is the wave number in any direction and $\widehat{E_{1}}$ is the spectral energy density at $k=1_{m}^{-1}$. Following Hogan and Kew (2005), for the idealized case where $g(x, y)$ is continuous at small scales and infinite in extent, its 2-D spectral density matrix $E_{2}\left(k_{x}, k_{y}\right)$ can be written as

$E_{2}(k)=\kappa \widehat{E_{1}} k^{-\beta_{1-\mathrm{D}}-1}$,

where $k=\sqrt{k_{x}^{2}+k_{y}^{2}}$ and $\kappa$ a constant. In general, a 2-D cloud layer of $\tau_{2-\mathrm{D}}$ (or $\mathrm{IWC}_{2-\mathrm{D}}$ ) is anisotropic and, in our case, the optical depth (or IWC) is gamma-distributed. Therefore, the 1-D power spectrum $E_{1}(k)$ seldom has the required spectral slope $\beta_{1-\mathrm{D}}$. In this context, a numerical method has to be developed to perform our objectives.

Let set $\widehat{Y_{2-\mathrm{D}}}$ be the 2-D Fourier transform of $Y_{2-\mathrm{D}}$, where $Y_{2-\mathrm{D}}$ can be $\tau_{2-\mathrm{D}}$ (or $\mathrm{IWC}_{2-\mathrm{D}}$ ) at a given cloudy layer. This quantity, estimated with the help of a direct 2-D fast Fourier transform algorithm can be written as follows:

$\widehat{Y_{2-\mathrm{D}}}(k)=E_{2-\mathrm{D}}(k) \exp \left(i \phi_{2-\mathrm{D}}(k)\right)$,

where $E_{2-\mathrm{D}}=\left|\widehat{Y_{2-\mathrm{D}}}\right|$ is the magnitude or spectral energy, $\phi_{2-\mathrm{D}}(k)$ are the phase angles and $k=\sqrt{k_{x}^{2}+k_{y}^{2}}$ is the absolute wave number. The cloud field domain is defined to measure $L_{x}$ and $L_{y}$ and they have spatial resolutions of $\Delta x$ and $\Delta y$. The resulting wave number for $E_{2-\mathrm{D}}$ ranges from $-K_{x}$ to $+K_{x}$ with a resolution of $\Delta k_{x}=1 / L_{x}$, where $K_{x}=1 / 2 \Delta x$. It is similar for $k_{y}$ direction.

Our objective is to modify spectral energy $E_{2 \text {-D }}(k)$ in such a way that the 1-D spectral slope value $\mu_{1-\mathrm{D}}$ estimated in one dimension from $Y_{2-\mathrm{D}}$ for $k \geq k_{\text {out }}\left(k_{\text {out }}=1 / L_{\text {out }}\right)$ satisfies the desired value $\beta_{1-\mathrm{D}}$ required by the user. Practically, $\mu_{1-\mathrm{D}}$ is defined as follows:

$\mu_{1-\mathrm{D}}=\left(\beta_{x}+\beta_{y}\right) / 2$,

where $\beta_{x}$ and $\beta_{y}$ are the 1-D spectral slope values of $\widehat{Y_{2-D}}$ estimated in the $x$ and $y$ directions respectively.

In order to conserve the spatial repartition of $Y_{2-D}$, we keep $\phi_{2 \text {-D }}(k)$ phase angles unchanged for all values of $k$. We also keep unchanged $E_{2-\mathrm{D}}(k)$ for $k<k_{\text {out }}$. For $k \geq k_{\text {out }}$, two 2D matrix $\mathbf{E}_{2-\mathrm{D}}^{*}(k)$ and $\mathbf{E}_{2-\mathrm{D}}^{* *}(k)$ can be computed. $\mathbf{E}_{2-\mathrm{D}}^{*}(k)$ is based on Eq. (18) and is defined as follows:

$\mathbf{E}_{2-\mathrm{D}}^{*}(k)=\frac{k^{\left(-\beta_{1-\mathrm{D}}-1\right)}}{\overline{k_{\mathrm{out}}^{\left(-\beta_{1-\mathrm{D}}-1\right)}}} \overline{E_{2-\mathrm{D}}}\left(k=k_{\mathrm{out}}\right)$ whereas $\mathbf{E}_{2-\mathrm{D}}^{* *}(k)$ is defined as follows:

$\mathbf{E}_{2-\mathrm{D}}^{* *}(k)=\frac{E_{2-\mathrm{D}}(k)}{\overline{E_{2-\mathrm{D}}}(k)} \frac{k^{\left(-\beta_{1-\mathrm{D}}-1\right)}}{\overline{k_{\text {out }}^{\left(-\beta_{1-\mathrm{D}}-1\right)}}} \overline{E_{2-\mathrm{D}}}\left(k=k_{\text {out }}\right)$,

where $\bar{X}$ is the mean of variable $X$. If the degree of anisotropy of $Y_{2-D}$ is small, such as for stratocumulus and cumulus, we use $\mathbf{E}_{2-\mathrm{D}}^{*}(k)$ and if not, such as for cirrus clouds, $\mathbf{E}_{2-\mathrm{D}}^{* *}(k)$. Nonetheless, the user can also choose one of these methods.

Finally, the new 2-D local optical depth (or the 2-D new ice water content $\left.Y_{2-\mathrm{D}}^{\mathrm{new}}\right)$ at the given cloud layer is computed by keeping the real part of the inverse 2-D fast Fourier transform of the new quantity:

$$
\begin{aligned}
\widehat{Y_{2-\mathrm{D}}^{\text {new }}}(k)= & \mathbf{E}_{2-\mathrm{D}}^{*}(k) \exp \left(i \phi_{2-\mathrm{D}}(k)\right) \text { or } \widehat{Y_{2-\mathrm{D}}^{\mathrm{new}}}(k)= \\
& \mathbf{E}_{2-\mathrm{D}}^{* *}(k) \exp \left(i \phi_{2-\mathrm{D}}(k)\right) .
\end{aligned}
$$

But as a result, the distribution of $Y_{2-\mathrm{D}}^{\text {new }}$ is not the same $Y_{2-D}$ at the given cloud layer, and the equality between the estimated spectral slope $\mu_{1-\mathrm{D}}$ of $Y_{2-\mathrm{D}}^{\text {new }}$ and the required values $\beta_{1-\mathrm{D}}$ is not always guaranteed. Therefore, we have to redo an "amplitude adaptation", as explained in Venema et al. (2006), and iterate the process explained in this section by changing the value of $\beta_{1-D}$ in Eqs. (21) or (22), until the estimated value $\mu_{1-\mathrm{D}}$ reaches the required value within a few percent.

\subsubsection{Implementation}

We describe here the part of the 3DCLOUD algorithm that establishes the cloud field mean optical depth $\bar{\tau}(\overline{\mathrm{IWC}})$, the inhomogeneity parameter $\rho_{\tau}$ (or $\rho_{\text {IWC }}$ ) and the spectral exponent $\beta_{1-\mathrm{D} \text {. }}$

For stratocumulus and cumulus clouds, the algorithm is the following.

1. Transformation of $\operatorname{LWC}(x, y, z)$ to $\tau_{3-\mathrm{D}}^{\prime}(x, y, z)$ with Eq. (10). Effective radius can be set to $10 \mu \mathrm{m}$ for example.

2. Application of the algorithm explained in Sect. 2.2.2 in order to constrain $\beta_{1-D}$ of each cloudy layer of $\tau_{3-\mathrm{D}}^{\prime}(x, y, z)$. We obtain $\tau_{3-\mathrm{D}}^{\prime \prime}(x, y, z)$.

3. Computation of optical depth $\tau^{\prime}(x, y)$ from $\tau_{3-\mathrm{D}}^{\prime \prime}(x, y, z)($ Eq. 11).

4. Transformation of $\tau^{\prime}(x, y)$ to $\tau^{\prime \prime}(x, y)$ with the help of the algorithm explained in Sect. 2.2.1 in order to constrain $\bar{\tau}$ and $\rho_{\tau}$ values.

5. Transformation of $\tau^{\prime \prime}(x, y)$ to $\tau^{\prime \prime \prime}(x, y)$ with the algorithm explained in Sect. 2.2.2 in order to $\operatorname{control} \beta_{1-\mathrm{D}}$ value.

6. Normalization of $\tau^{\prime \prime \prime}(x, y)$ to the required $\bar{\tau}$ value, in order to obtain $\tau_{3-\mathrm{D}}(x, y, z)$. 
For cirrus clouds, the algorithm is as follows.

1. Transformation of $\operatorname{IWC}(x, y, z)$ to $\operatorname{IWC}^{\prime}(x, y, z)$ with the algorithm explained in Sect. 2.2.1 in order to constrain $\overline{\mathrm{IWC}}$ and $\rho_{\text {IWC }}$ values.

2. Application of the algorithm explained in Sect. 2.2.2 in order to constrain $\beta_{1-\mathrm{D}}$ of each cloud layer of $\operatorname{IWC}^{\prime}(x, y, z)$. We obtain $\operatorname{IWC}^{\prime \prime}(x, y, z)$.

3. Transformation of $\operatorname{IWC}^{\prime \prime \prime}(x, y, z)$ to $\operatorname{IWC}_{3-\mathrm{D}}(x, y, z)$ with the algorithm explained in Sect. 2.2.1 in order to constrain $\overline{\mathrm{IWC}}$ and $\rho_{\text {IWC }}$ values.

\subsection{Differences between 3DCLOUD, IAFFT method and Cloudgen models}

Both IAAFT (Venema et al., 2006) and Cloudgen (Hogan and Kew, 2005) models are purely stochastic Fourier based approaches that are able to generate synthetic or surrogate cloud. On the contrary, 3DCLOUD solves, in a first step, basic atmospheric equations, in order to generate an intermediate cloud field. In its second step, as for both IAAFT and Cloudgen models, it uses Fourier tools (manipulation of energy and phase in frequency space) and amplitude adaptation (manipulation of distributions) in order to generate the final cloud field. IAAFT and Cloudgen are designed to simulate stratocumulus/cumulus fields for the first and cirrus fields for the second, when 3DCLOUD is able to simulate stratocumulus, cumulus and cirrus field within the same framework.

More specifically, the IAAFT method is designed to generate surrogate clouds having both the amplitude distribution and power of the original cloud (2-D LWC from 1-D LWP measurement, 3-D LWC from 2-D LWC fields or 3-D LWC from 3-D fields generated by LES). It needs LES inputs or measurements. As explained in Venema et al. (2006), stratocumulus often display beautiful cell structures, similar to Bénard convection, and LES clouds show such features. But their 3-D IAFFT surrogates show these much less and do not show fallstreak or a filamentous structure. Due to the specific manipulations of Fourier coefficients presented in the paper, we show that 3DCLOUD is able to simulate the cell structure of stratocumulus (see Figs. 7c and 8), the filamentous structure of cirrus (see Fig. 13) and the cirrus fallstreaks (see Figs. 14 and 15) relatively well. Moreover, the objective of 3DCLOUD is not to provide many surrogate clouds with the same amplitude distribution and power spectrum from an LES original cloud, but to provide 3-D LWC (or optical depth) with the required cloud coverage, the $-5 / 3$ spectral slope (often observed in real clouds), the mean value of the gamma distribution of the optical depth and the inhomogeneity parameter, all these parameters being very pertinent for radiative transfer.

Cloudgen is designed to simulate surrogate cirrus with the cirrus specific structural properties: fallstreak geometry and shear-induced mixing. It first generates a 3-D fractal field by performing an inverse 3-D Fourier transform on a matrix of simulated Fourier coefficients with amplitude consistent with observed 1-D spectra. Then random phases are generated for the coefficient allowing multiple cloud realizations with the same statistical properties. Horizontal slices from the domain are manipulated in turn to simulate horizontal displacement and to change the spectra with height. The final field is scaled to produce the observed mean and fractional standard deviation of ice water content. 3DCLOUD does not use a 3-D fractal field, but a 3-D IWC field simulated by the simplified atmospheric equation set. Therefore, cloud structures due to wind shear are physically obtained by taking into account the advection (a nonlinear term in momentum equation) rather than by a linear horizontal displacement of phase. Afterwards, in the current version of 3DCLOUD, for each level, 2-D horizontal slices of this 3-D IWC are manipulated in 2D Fourier domain in such a way that the Fourier coefficient amplitude is consistent with the 2-D spectra of the simulated IWC, with the constraint that the 1-D spectral slope is equal to $-5 / 3$ (this value can be change easily in future version of 3DCLOUD). At each level, the 2-D phase for the coefficient is kept unchanged. Finally, the mean value of the 3-D IWC and the inhomogeneity parameter are adjusted. As explained by Hogan and Kew (2005), it is difficult with Cloudgen to generated anisotropic cirrus structure such as roll-like structure near cloud top. 3DCLOUD, using physically based equations, allows simulating such kinds of anisotropy, as for example, 3DCLOUD Kelvin-Helmholtz wave breaking (see Fig. 14).

\section{Comparison between 3DCLOUD and large-eddy simulation (LES) outputs}

The objective of this section is to check that the basic atmospheric equations used in 3DCLOUD (see Sect. 2.1.1) are solved correctly. We compare 3DCLOUD and LES outputs found in the scientific literature for marine stratocumulus, cumulus and cirrus regimes. Note that assimilation techniques of meteorological profiles and of cloud coverage (see Sect. 2.1.2) are not used here, except in Sect. 3.4.

The test cases come from the output LES numerical files provided by the Global Water and Energy Experiment (GEWEX) Cloud System Studies (GCSS) Working Group 1 (WG1) and Working Group 2 (WG2), easily downloadable from the web. They are often used as a benchmark. We choose the DYCOMS2-RF01 case (the first Research Flight of the second Dynamics and Chemistry of Marine Stratocumulus) for the marine stratocumulus regime (Stevens et al., 2005), the BOMEX case (Barbados Oceanographic and Meteorological Experiment) for the shallow cumulus regime (Siebesma et al., 2003), and the ICMCP case (Idealized Cirrus Model Comparison Project) for cirrus regimes (Starr, 2000). 

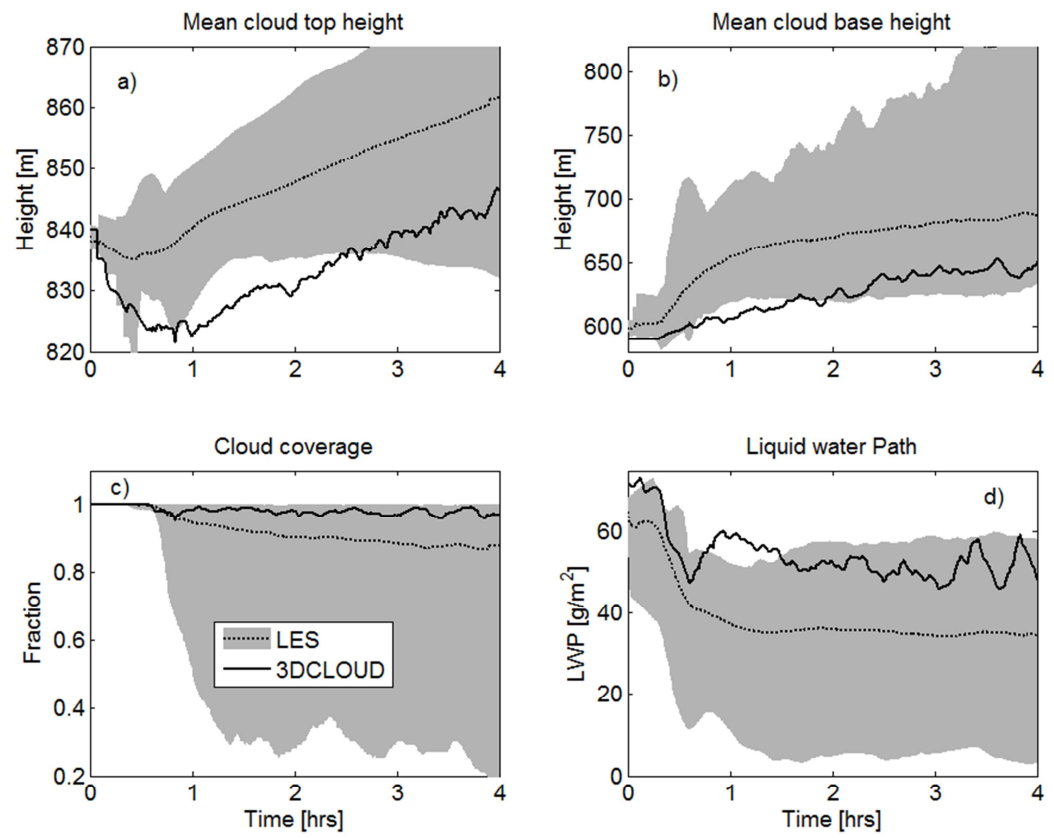

Figure 2. Time series of (a) the mean cloud top height, (b) the mean cloud base height, (c) the cloud coverage, and (d) the liquid water path. The DYCOM2-RF01 case is displayed.The solid lines indicate 3DCLOUD results. The dotted lines indicate a mean over all LES results. The light shading around this mean delimits the maximum and minimum values within the master ensemble at any given time.

\subsection{DYCOMS2-RF01 (GCSS-WG1) case}

We remember briefly the conditions of simulations and configurations, explained in detail in Stevens et al. (2005). A $4 \mathrm{~h}$ simulation on a horizontal grid of 96 by 96 points with $35 \mathrm{~m}$ spacing between grid nodes was required. Vertical spacing was required to be $5 \mathrm{~m}$ or less. In 3DCLOUD, we thus set $N_{x}=N_{y}=96$ and $N_{z}=240, L_{x}=L_{y}=3.5 \mathrm{~km}$ and $L_{z}=1200 \mathrm{~m}$, so that $\Delta x=\Delta y \approx 36.5 \mathrm{~m}$ and $\Delta z=5 \mathrm{~m}$. Initial profiles of the liquid water potential temperature $\theta_{1}$ and of the total water mixing ratio $q_{\mathrm{t}}$ are $\theta_{\mathrm{l}}=289.0 \mathrm{~K}$ and $q_{\mathrm{t}}=9.0 \mathrm{~g} \mathrm{~kg}^{-1}$ if $z \leq z_{\mathrm{i}}$ and $\theta_{\mathrm{l}}=297.5+\left(z-z_{\mathrm{i}}\right)^{1 / 3} \mathrm{~K}$ and $q_{\mathrm{t}}=1.5 \mathrm{~g} \mathrm{~kg}^{-1}$ if $z>z_{\mathrm{i}}$. Other required forcings include geostrophic winds $\left(U_{\mathrm{g}}=7 \mathrm{~m} \mathrm{~s}^{-1}\right.$ and $\left.V_{\mathrm{g}}=-5.5 \mathrm{~m} \mathrm{~s}^{-1}\right)$, divergence of the large-scale winds $\left(D=3.75 \times 10^{-6} \mathrm{~s}^{-1}\right)$, surface sensible heat flux $\left(15 \mathrm{~W} \mathrm{~m}^{-2}\right)$ and surface latent heat flux $\left(115 \mathrm{~W} \mathrm{~m}^{-2}\right)$. The momentum surface fluxes where the total momentum is specified by setting $u^{*}=0.25 \mathrm{~m} \mathrm{~s}^{-1}$ and the radiation schemes is based on a simple model of the net long wave radiative flux (see Eqs. 3 and 4 in Stevens et al., 2005).

Figure 2 shows the evolution of the mean cloud top height, the mean cloud base height, the cloud coverage and the liquid water path from the master ensemble and for 3DCLOUD during the $4 \mathrm{~h}$ simulations. Even though we can notice slight discrepancies between 3DCLOUD and master ensemble results in the first $2 \mathrm{~h}$ ("spin-up" period), 3DCLOUD results are quite consistent with master ensemble results, especially at the end of the simulation. Nevertheless, 3DCLOUD tends to generate a lower cloud height than the mean results with a higher liquid water path.

Figure 3 shows the mean profiles averaged over the fourth hour of the long wave net flux, the liquid water potential temperature, the total water mixing ratio, the liquid water mixing ratio, the horizontal velocity components, and the air density. Even though the 3DCLOUD long wave net flux is smaller compared to master ensemble, again 3DCLOUD results are quite consistent with other results for all the variables.

\subsection{BOMEX (GCSS-WG1) case}

For the BOMEX case (Siebesma et al., 2003), a $6 \mathrm{~h}$ simulation on a horizontal grid of 64 by 64 points with $100 \mathrm{~m}$ spacing between grid node was required. Vertical spacing was required to be $40 \mathrm{~m}$. In 3DCLOUD, we set thus $N_{x}=N_{y}=64$ and $N_{z}=76$ with $L_{x}=L_{y}=6.4 \mathrm{~km}$ and $L_{z}=2980 \mathrm{~m}$, so $\Delta x=\Delta y=100 \mathrm{~m}$ and $\Delta z \approx 39.2 \mathrm{~m}$. Initial profiles of the liquid water potential temperature $\theta_{1}$ and the total water mixing ratio $q_{\mathrm{t}}$ and the other requirement including geostrophic winds, divergence due to the subsidence, surface sensible heat flux, surface latent heat flux, momentum surface fluxes, moisture large scale horizontal advection term and long wave radiative cooling (radiative effects due to the presence of clouds are neglected) are presented in Appendix B in Siebesma et al., 2003).

Figure 4 shows the evolution of the cloud coverage and the liquid water path from the master ensemble and for 3DCLOUD during the $6 \mathrm{~h}$ simulation. We can notice 

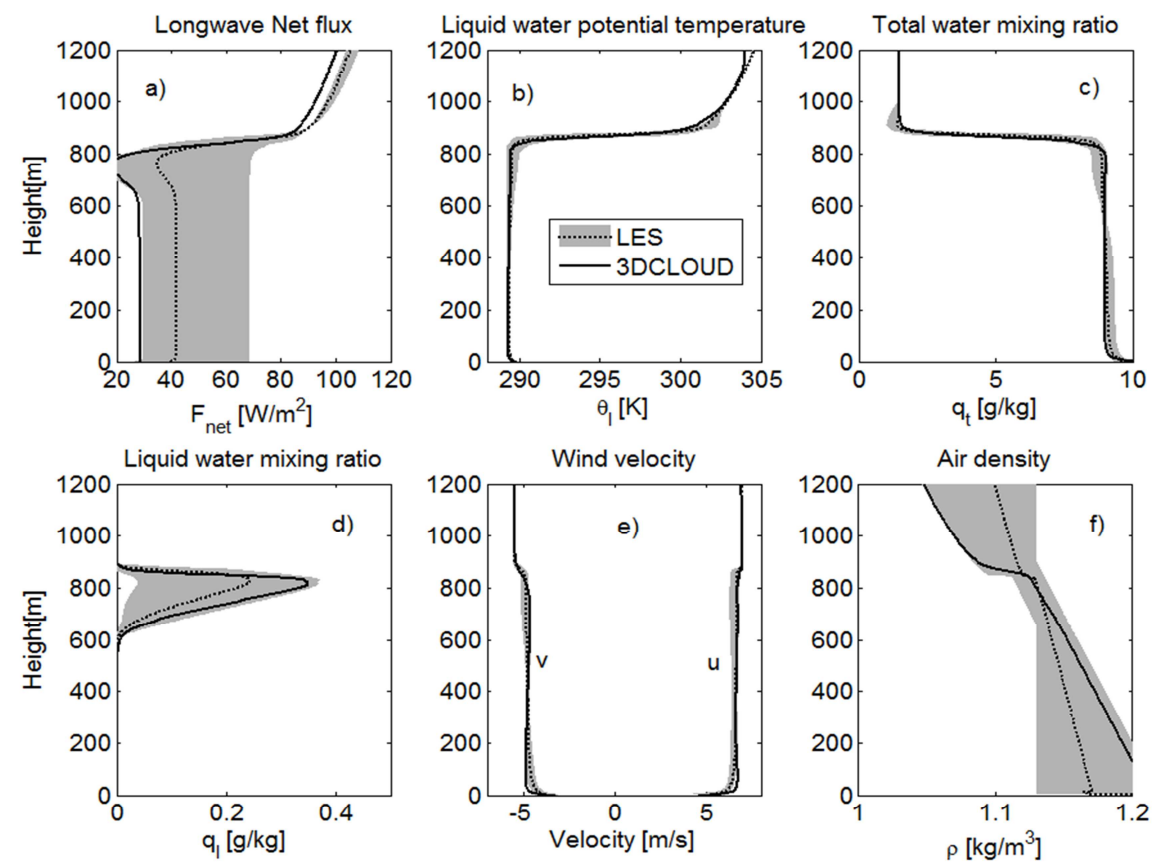

Figure 3. Mean profiles averaged over the fourth hour of (a) the long wave net flux, (b) the liquid water potential temperature, (c) the total water mixing ratio, (d) the liquid water mixing ratio, (e) the horizontal velocity components, and (f) the air density. The DYCOM2-RF01 case is displayed. The solid lines indicate 3DCLOUD results. The dotted lines indicate a mean over all LES results. The light shading around this mean delimits by the maximum and minimum values within the master ensemble at any given height.
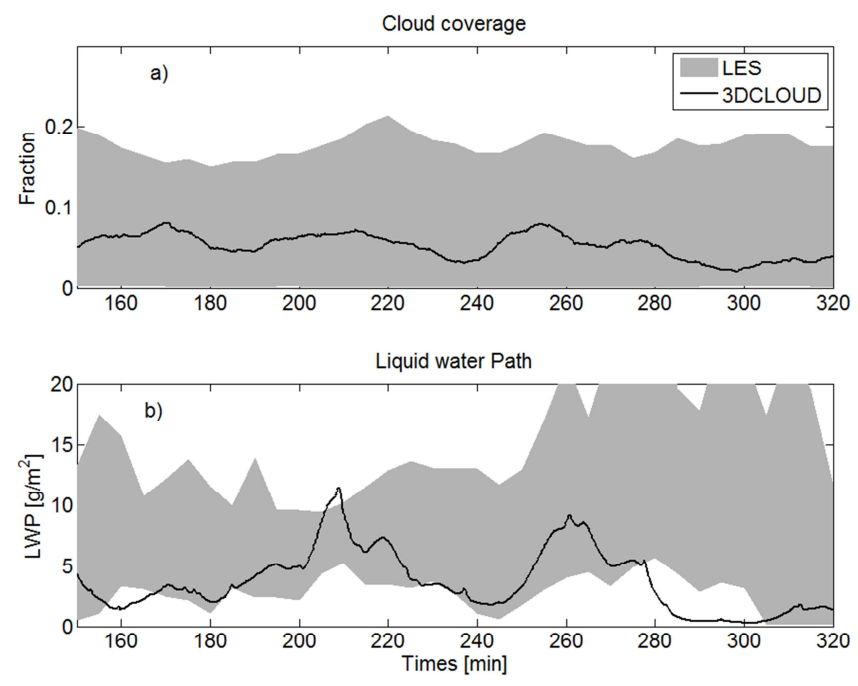

Figure 4. Time series of (a) the cloud coverage and (b) the liquid water path. The BOMEX case is displayed. The solid lines indicate 3DCLOUD results. The light shading delimits the maximum and minimum values within the master ensemble at any given time.

the small value of the cloud coverage (less than $10 \%$ ). 3DCLOUD results are quite consistent with the master ensemble results, even if the simulated 3DCLOUD liquid water path (LWP) may be too low at the end of the simulation.
Figure 5 shows mean profiles, averaged over the fifth hour of the cloud coverage, potential temperature, water vapour mixing ratio, liquid water mixing ratio, horizontal velocity components, and air density. The 3DCLOUD results are again quite consistent with the master ensemble results. We note, however, that the 3DCLOUD cloud coverage and liquid water mixing ratio are smaller at all heights. We also see small differences (less than $1 \mathrm{~m} \mathrm{~s}^{-1}$ ) for the wind velocity below $500 \mathrm{~m}$, and for the potential temperature (less than $1 \mathrm{~K}$ ) and water vapour mixing ratio (less than $1 \mathrm{~g} \mathrm{~kg}^{-1}$ ) for altitudes $1800 \mathrm{~m}$.

\subsection{ICMCP (GCSS-WG2) case}

For the cirrus case detailed in Starr et al. (2000), the baseline simulations include night-time "warm" cirrus and "cold" cirrus cases where cloud top initially occurs at $-47^{\circ} \mathrm{C}$ and $-66^{\circ} \mathrm{C}$, respectively. The cloud is generated in an ice super-saturated layer with a geometric thickness around $1 \mathrm{~km}$ $(120 \%$ in $0.5 \mathrm{~km}$ layer) and with a neutral ice pseudoadiabatic thermal stratification. Cloud formation is forced via an imposed diabatic cooling over a $4 \mathrm{~h}$ time span followed by a $2 \mathrm{~h}$ dissipation stage without cooling. All models simulate radiative transfer, contrary to 3DCLOUD. In 3DCLOUD, we set $N_{x}=N_{y}=60$ and $N_{z}=140$ with $L_{x}=L_{y}=6.3 \mathrm{~km}$ and $L_{z}=14 \mathrm{~km}$, so that $\Delta x=\Delta y=105 \mathrm{~m}$ and $\Delta z \approx 100 \mathrm{~m}$.

Figure 6 shows the evolution of the ice water path (IWP) from the master ensemble and for 3DCLOUD during the 

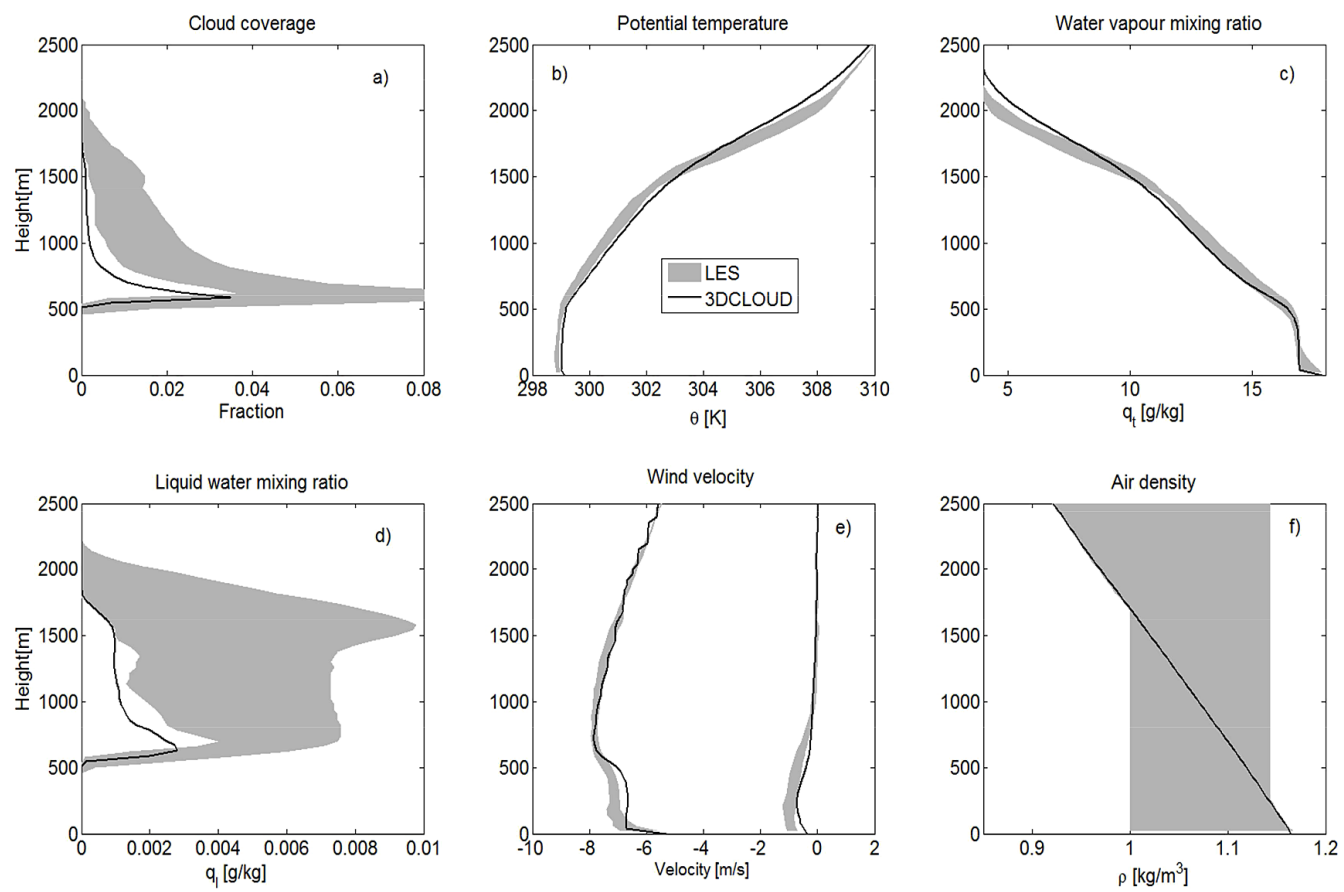

Figure 5. Mean profiles averaged over the fifth hour of (a) cloud coverage, (b) the potential temperature, (c) the water vapour mixing ratio, (d) the liquid water mixing ratio, (e) the horizontal velocity components, and (f) the air density. The BOMEX case is displayed. The solid lines indicate 3DCLOUD results. The light shading delimits the maximum and minimum values within the master ensemble at any given height.

$6 \mathrm{~h}$ simulation. In a general way, most of the tested models and 3DCLOUD have similar behaviour: indeed, the IWP increases during the first $4 \mathrm{~h}$ simulation (cirrus formation due to imposed cooling) and decreases after (cirrus dissipation due to non-imposed cooling). The IWP range of the tested models is very large (factor of 10), but we can notice that 3DCLOUD behaviour is closer to bulk microphysics models behaviours, especially for "warm cirrus".

For "cold" cirrus, the 3DCLOUD IWP is smaller than most participating models during all the simulation duration. It is probably because 3DCLOUD does not account for the radiative transfer, as opposed to the participating models. Indeed, neglecting cirrus top cooling due to radiative processes restricts the formation of thin "cold" cirrus. This radiative diabatic effect is probably less important for the "warm" cirrus because the latent heat diabatic effect is larger.

\subsection{Comparison between 3DCLOUD and BRAMS for the DYCOM2-RF01 case}

In order to underscore differences between 3DCLOUD and LES for comparable scenes, we choose again the well documented DYCOMS2-RF01 case. Snapshots can be found, for example, in Stevens et al. (2005) and in Yamaguchi and Feingold (2012). We performed the $4 \mathrm{~h}$ simulations of the DYCOMS2-RF01 case with 3DCLOUD and with the Brazilian Regional Atmospheric Modelling System (BRAMS v4) model (Pielke at al., 1992; Cotton et al., 2003). BRAMS simulations were provided by G. Penide (Penide et al., 2010). The BRAMS model is constructed around the full set of nonhydrostatic, compressible equations. The cloud microphysics parameterization is based on a two-moment scheme (Meyers et al., 1997). Subgrid scale fluxes are modelled following Deardroff (1980). The base calculations are performed on a $100 \times 100 \times 100$ point mesh with a step time of $0.3 \mathrm{~s}$.

Figure 7 shows the instantaneous cloud-field snapshots of the pseudo albedo (see definition in Sect. 4) at 4 hours simulated by (a) the UCLA-0 model (picture taken from Stevens et al., 2005), (b) the BRAMS model, both configured following the DYCOMS2-RF01 case (Stevens et al., 2005) and (c) from 3DCLOUD with assimilation of meteorological profiles based on the DYCOMS-RF01 case. Both BRAMS and 3DCLOUD cases are drawn from simulations where $\Delta_{x}=\Delta_{y}=40 \mathrm{~m}$ and $\Delta_{z}=12 \mathrm{~m}$. These three snapshots of cloud fields are characterized by closed cellular convection with large cloud cover, as argued in Yamaguchi and Feingold (2012), who did simulation of DYCOMS-RF01 case with the LES mode of the Advanced Research weather research and forcasting (WRF) model. Figure 7 also shows the power spectra computed following the $x$ and $y$ directions and then averaged, for BRAMS and 3DCLOUD optical depth fields. The 3DCLOUD optical depth spectral slope is close to $-5 / 3$ in the $\left[L_{\text {out }}: 1 /(2 \Delta x)\right] \mathrm{m}^{-1}$ wave number range, as expected, because of the statistical adjustment 

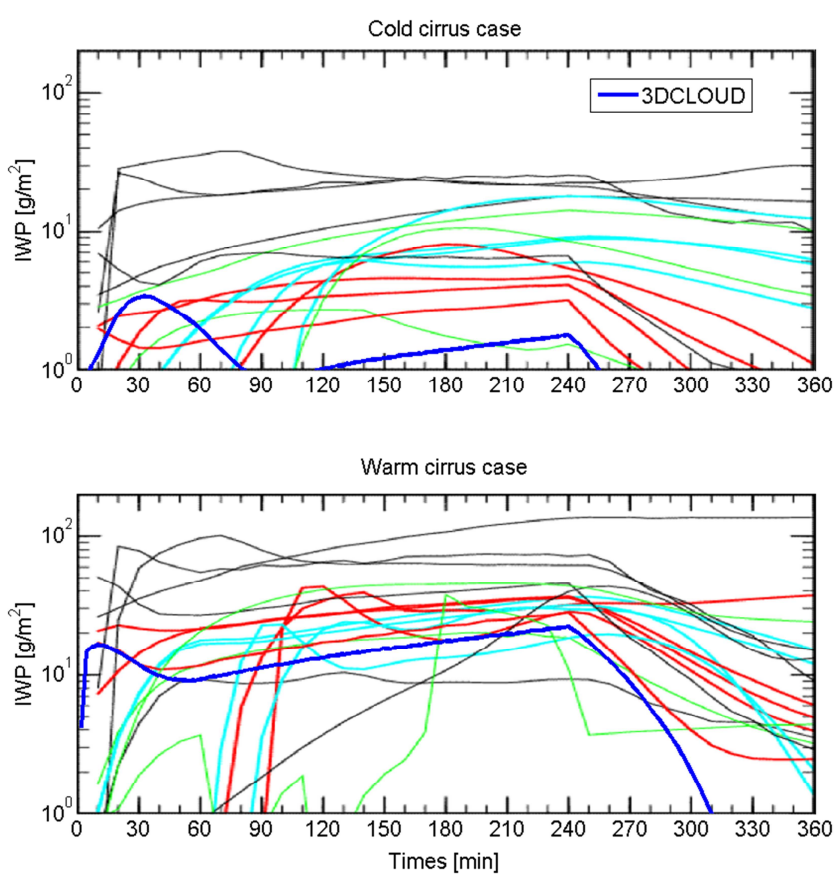

Figure 6. Time series of vertically integrated ice water path (IWP) from different cirrus models, which participated in the Idealized Cirrus Model Comparison Project and from the 3DCLOUD model (thick blue lines). The upper panel is for the cold cirrus case and the bottom one is for the warm cirrus case. Cyan line represents models with bin microphysics, red line models with bulk microphysics, green line single column models, and thin black lines models with heritage in the study of deep convection or boundary layer clouds. This figure is made from the one taken from Starr et al. (2000) and Yang et al. (2012).

performed in the second step of the 3DCLOUD algorithm. By contrast, the BRAMS optical depth spectral slope is close to $-5 / 3$ only in the $\left[2 \times 10^{-3}: 5 \times 10^{-3} \approx 1 /(5 \Delta x)\right] \mathrm{m}^{-1}$ wave number range. Depending on their degree of sophistication, LES do not always guarantee cloud invariant scale properties at the larger wave numbers. Indeed, Bryan et al. (2003) have shown, that for the finite-difference model, the vertical wind velocity spectral slope is steeper than $-5 / 3$ for scales shorter than $6 \Delta x$. Table 1 shows the computation performance of 3DCLOUD and BRAMS. For this specific case, 3DCLOUD simulation is 30 times faster than BRAMS simulation.

\section{Examples of 3DCLOUD possibilities}

In this section, we present cloud fields generated by 3DCLOUD with the assimilation of idealized meteorological profiles and fractional cloud coverage defined by the user. We also show the effect of the outer scale $L_{\text {out }}$ and the inhomogeneity parameter of optical depth $\rho_{\tau}$ on the generated optical depth field. We also give an example of cirrus clouds with fallstreaks. In order to have a spatial representation of the clouds as seen from above, we choose to show the socalled pseudo-albedo $\alpha$ defined as follows:

$\alpha=\frac{(1-g) \tau}{2+(1-g) \tau}$,

where the asymmetry parameter $g$ is set to 0.86 and $\tau$ is the optical depth.

\subsection{Stratocumulus and cumulus fields with assimilation of meteorological profiles based on DYCOMS2-RF01 and BOMEX cases}

We choose to simulate stratocumulus and cumulus LWC in the context of DYCOMS2-RF01 and BOMEX cases. With this aim, we assimilate temperature and humidity initial profiles for stratocumulus and cumulus given by Stevens et al. (2005) and Siebesma et al. (2003), respectively. However, in order to mimic the sensible and latent heat, these profiles have to be slightly modified. At sea surface $(z=0)$, for stratocumulus (DYCOMS2-RF01 case), the liquid potential temperature and total mixing ratio are set to $290 \mathrm{~K}$ and $10 \mathrm{~g} \mathrm{~kg}^{-1}$, respectively (instead of $289 \mathrm{~K}$ and $9 \mathrm{~g} \mathrm{~kg}^{-1}$, respectively). For cumulus (BOMEX case), the liquid potential temperature is set to $299.7 \mathrm{~K}$ instead of $298.7 \mathrm{~K}$. In addition, wind profiles assimilated by 3DCLOUD are those computed by the master ensemble at the end of simulation.

\subsubsection{Effects of numerical spatial resolution}

The effects of the numerical spatial resolution on 3DCLOUD simulations are presented herein. Figure 8 shows pseudoalbedo and cross sections of the vertical velocity and cloud water at the end of the simulation for the stratocumulus case based on the DYCOMS2-RF01 experiment. It also shows the mean profiles of potential temperature, liquid water mixing ratio, horizontal velocity components, and vapour water mixing ratio for different numerical spatial resolutions $\Delta x=\Delta y=200 \mathrm{~m}, 100 \mathrm{~m}, 50 \mathrm{~m}$ and $25 \mathrm{~m}$. Horizontal extensions $L_{x}=L_{y}$ are set to $10 \mathrm{~km}$ and vertical resolution $\Delta z$ to $24 \mathrm{~m}$ for all simulations. Figure 9 is the same as Fig. 8, but for the cumulus case with assimilation of meteorological profiles based on the BOMEX case. The vertical resolution $\Delta z$ is set to $38.5 \mathrm{~m}$ in this last case.

It is obvious that change in the horizontal mesh leads to a more pleasant and detailed flow visualization but there is no significant impact on the mean statistics of the simulated temperature vertical profile, water vapour mixing ratio and wind velocity. The water mixing ratio simulated by 3DCLOUD for the DYCOMS2-RF01 case is very close to the mean profile averaged over the fourth hour and provided by the master ensemble, even if the vertical resolution used in this section is only $\Delta z=38.5 \mathrm{~m}$ compared to $\Delta z=5 \mathrm{~m}$ in Sect. 3.1. For the BOMEX case, the water mixing ratio simulated by 3DCLOUD changes as a function of the numerical 


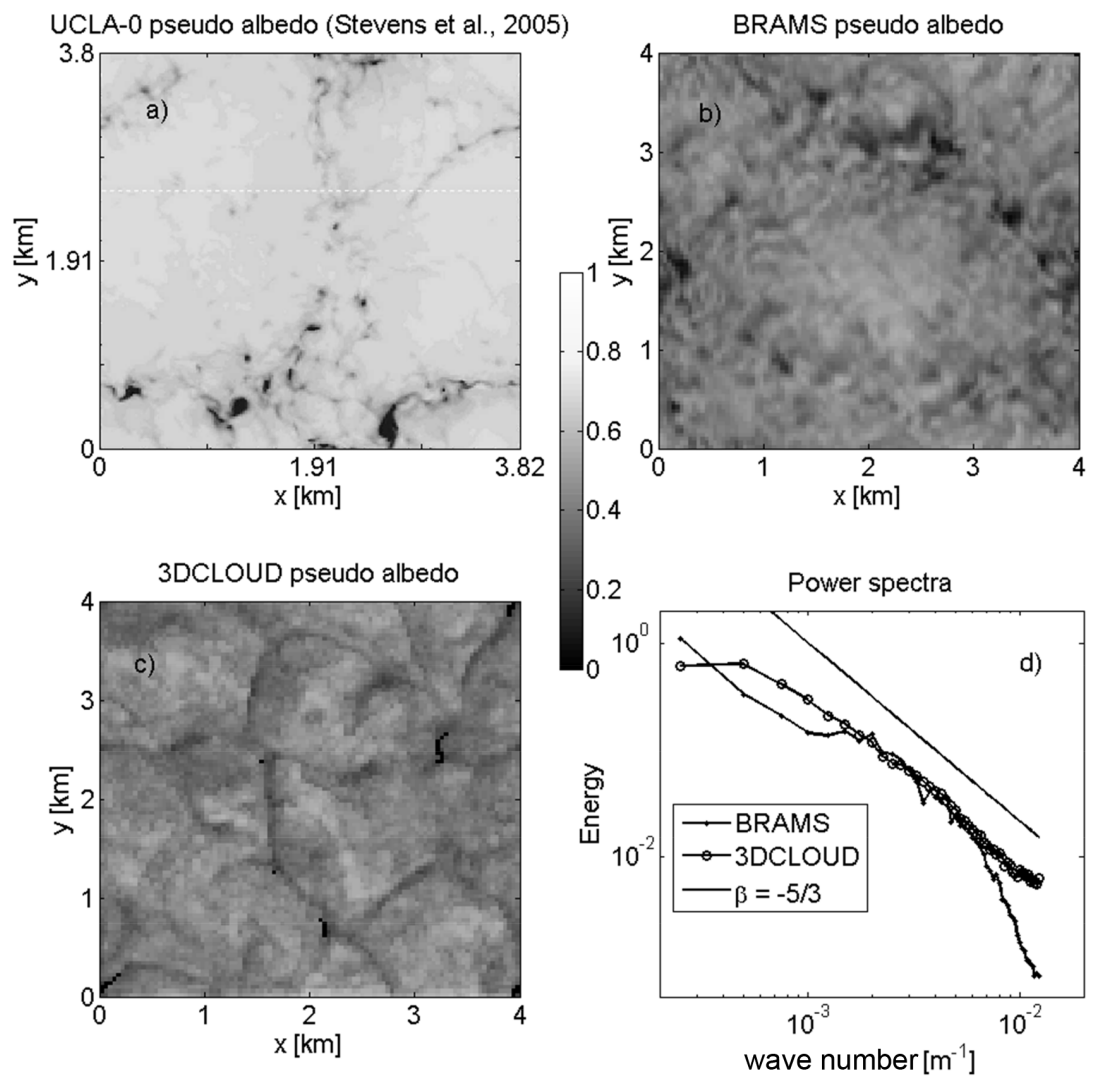

Figure 7. The instantaneous cloud-field snapshots of the pseudo albedo at 4 hours simulated by (a) the UCLA-0 model (picture taken from Stevens et al., 2005), (b) the BRAMS model, both configured following the DYCOMS2-RF01 case (Stevens et al., 2005) and (c) from 3DCLOUD with assimilation of meteorological profiles based on the DYCOMS-RF01 case. The UCLA-0 field is drawn from simulation where $N_{x}=N_{y}=192$ and $\Delta_{x}=\Delta_{y}=20 \mathrm{~m}$. Both BRAMS and 3DCLOUD are drawn from simulations where $N_{x}=N_{y}=N_{z}=100$, $\Delta_{x}=\Delta_{y}=40 \mathrm{~m}$ and $\Delta_{z}=12 \mathrm{~m}$. Note that the 3DCLOUD field is obtained at the second step of the algorithm, with the inhomogeneity parameter $\rho_{\tau}=0.3$, mean optical depth $\bar{\tau}=10$ and $L_{\text {out }}=2 \mathrm{~km}$. (d) is the optical depth power spectra computed following the $x$ and the $y$ directions and then averaged, for BRAMS (points) and 3DCLOUD (circles). A theoretical power spectrum with spectral slope $\beta=-5 / 3$ is added (black line).

spatial resolution. This behaviour is quite understandable as results drawn on Figs. 8 and 9 are snapshots at the end of the 3DCLOUD simulation and not average results over $1 \mathrm{~h}$ as done on Figs. 3 and 5. Moreover, BOMEX meteorological conditions cause time dependent cumulus fields, contrary to DYCOMS2 meteorological conditions that cause more stationary stratocumulus fields.

In addition, it is expected for the BOMEX case, that cloud spacing converges at high spatial resolution. In order to investigate it, we defined an estimator of the cloud spacing called the mean distance $D_{\text {mean }}$. To compute $D_{\text {mean }}$, the 3D LWC is vertically projected on the 2-D $x-y$ plan in order to obtain the 2-D binary image of the cloud coverage with free cloud areas set to 0 and cloudy areas set to 1 . Then we compute the mean distance between the cloud cell for the $x$ and $y$ directions to obtain $D_{\text {mean. }}$. Figure 10 shows time series of $D_{\text {mean }}$ for different horizontal spatial resolution $(\Delta x=\Delta y=192,100,50,25,12.5$ and $8.3 \mathrm{~m})$ with a constant vertical resolution $(\Delta z=38.5 \mathrm{~m})$, for cumulus cloud fields simulated by 3DCLOUD after assimilation of the BOMEX case meteorological profiles. The main difference between these simulations and the BOMEX case simulation is the smaller horizontal extension $L_{x}=L_{y}=5 \mathrm{~km}$ instead of $10 \mathrm{~km}$ in order to access high numerical spatial resolution $\Delta x=8.3 \mathrm{~m}\left(N_{x}=N_{y}=600, N_{Z}=70\right)$. Cumulus clouds appear 10 to $20 \mathrm{~min}$ after the beginning of the simulation. After $1 \mathrm{~h}$ of simulation, $D_{\text {mean }}$ is relatively constant with time, meaning that 3DCLOUD has converged. The mean distance averaged over the last half-hour of the $2 \mathrm{~h}$ simulation $\overline{D_{\text {mean }}}$, is also presented in Fig. 10 as a function of the numerical spatial resolution $\Delta x . \overline{D_{\text {mean }}}$ is relatively constant for a spatial resolution $\Delta x$ smaller than $20 \mathrm{~m}$, showing that BOMEX cloud spacing converges for spatial resolution close to $\Delta x=10 \mathrm{~m}$, a value smaller than $\Delta x=25 \mathrm{~m}$ used in Fig. 9d. 
Table 1. Time step, process time for one time step and process time for $2 \mathrm{~h}$-simulation with 3DCLOUD model, as a function of the numerical resolution. DYCOMS2-RF01 and BOMEX cases are presented. A comparison between 3DCLOUD and BRAMS LES computation time for a specific DYCOMS2-RF01 case is added. 3DCLOUD (Matlab code) runs on a personal computer with Intel Xeon E5520 (2.26 GHz) and BRAMS (Fortran code) runs on a PowerEdge R720 with Intel Xeon E5-2670 $(2.60 \mathrm{GHz})$, both of them having a single-processor configuration.

\begin{tabular}{llllll}
\hline Study case & $\begin{array}{l}\text { Point mesh } \\
N_{x} \times N_{y} \times N_{z}\end{array}$ & $\begin{array}{l}\text { Horizontal } \\
\text { numerical } \\
\text { resolution } \\
\Delta x[\mathrm{~m}]\end{array}$ & Time step [s] & $\begin{array}{l}\text { Process time } \\
{[\mathrm{s}]}\end{array}$ & $\begin{array}{l}\text { Process } \\
\text { time for 2 } \\
\text { simulation }\end{array}$ \\
& & 200 & 10 & 0.4 & 290 \\
\hline DYCOMS2- & $50 \times 50 \times 50$ & 7 & 1.3 & 1340 \\
RF01 & $100 \times 100 \times 50$ & 100 & 5 & 5 & 7200 \\
& $200 \times 200 \times 50$ & 50 & 3 & 18 & 43200 \\
\hline BOMEX & $400 \times 400 \times 50$ & 25 & 30 & 0.7 & 170 \\
& $100 \times 100 \times 70$ & 100 & 25 & 2.5 & 720 \\
& $200 \times 200 \times 70$ & 50 & 20 & 10 & 3600 \\
& $400 \times 400 \times 70$ & 25 & 14 & 40 & 20600 \\
\hline DYCOMS2- & $100 \times 100 \times 100$ & 40 & 13 & 2.7 & 1500 \\
RF01 & $100 \times 100 \times 100$ & 40 & 0.3 & 2 & 48600 \\
3DCLOUD & & & & & \\
BRAMS & & & & & \\
\hline
\end{tabular}
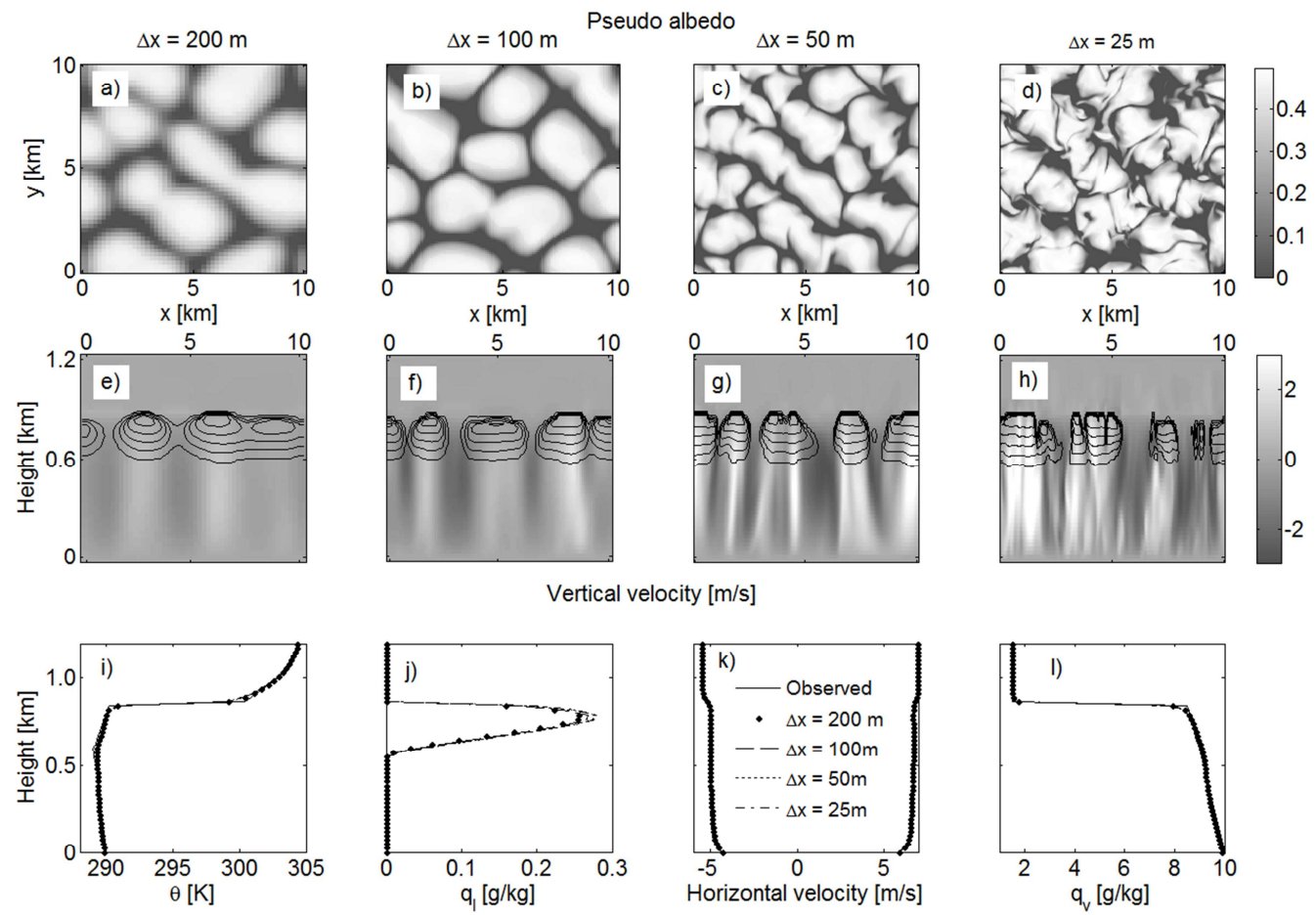

Figure 8. (a), (b), (c) and (d) pseudo albedo and (e), (f), (g), and (h) cross sections of the vertical velocity (shaded) and the cloud water (contoured), at the end of simulation, for the stratocumulus simulated by 3DCLOUD with assimilation of meteorological profiles based on the DYCOMS2-RF01 case. Different numerical spatial resolutions are presented with $\Delta x=\Delta y$ : (a) and (e) $\Delta x=200 \mathrm{~m}$, (b) and (f) $\Delta x=100 \mathrm{~m}$, (c) and (g) $\Delta x=50 \mathrm{~m}$ and (d) and (h) $\Delta x=25 \mathrm{~m}$. (i), (j), (k) and (l) mean profiles of the potential temperature, the liquid water mixing ratio, the horizontal velocity components, and the vapour water mixing ratio. The solid lines indicate meteorological profiles based on DYCOMS2-RF01 case and assimilated by 3DCLOUD. Points, dotted lines, dashed lines and dash-dot lines indicate 3DCLOUD results at the end of simulation for the different numerical spatial resolution $\Delta x=200 \mathrm{~m}, \Delta x=100 \mathrm{~m}, \Delta x=50 \mathrm{~m}$ and $\Delta x=25 \mathrm{~m}$, respectively. Number of iterations is 700 . 

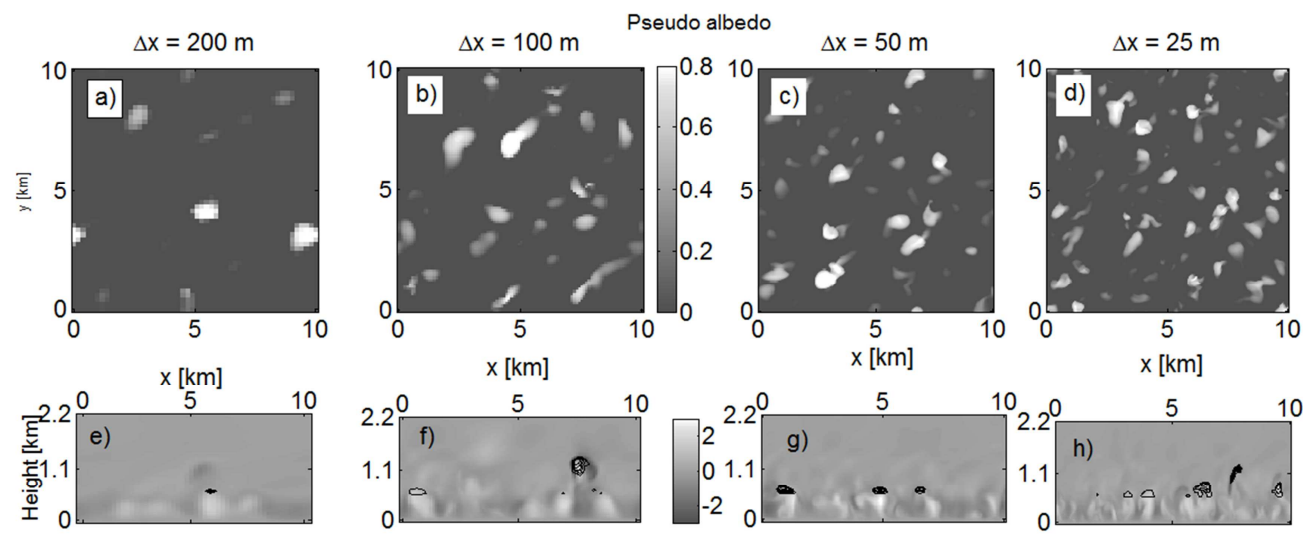

Vertical velocity $[\mathrm{m} / \mathrm{s}]$
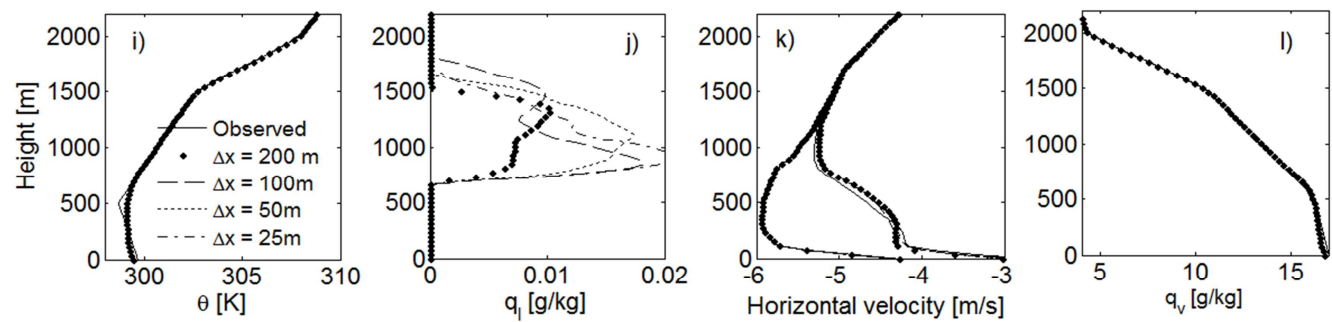

Figure 9. Same as Fig. 8, for cumulus cloud simulated by 3DCLOUD with assimilation of meteorological profiles based on the BOMEX case. We let 3DCLOUD iterating until $2 \mathrm{~h}$-simulation is done.

Table 1 shows the time step, process time for one time step and process time for $2 \mathrm{~h}$-simulation with 3DCLOUD model, as a function of the numerical resolution. DYCOMS2-RF01 and BOMEX cases are presented. The process time for $2 \mathrm{~h}$ simulation is indicated because 3DCLOUD algorithm convergence is achieved after $2 \mathrm{~h}$ (or less) of simulation for stratocumulus, cumulus and cirrus regimes (see Fig. 10 for cumulus case). For both cases, the smaller the spatial resolution, the smaller the step time and the larger the process time. A comparison between 3DCLOUD and BRAMS LES computation time for a specific DYCOMS2-RF01 case is added (see Sect. 3.4). For this specific case, 3DCLOUD simulation is 30 times faster than BRAMS simulation. Note that 3DCLOUD (Matlab code) runs on a personal computer with Intel Xeon E5520 (2.26 GHz) and BRAMS (Fortran code) runs on a PowerEdge R720 with Intel Xeon E5-2670 $(2.60 \mathrm{GHz})$, both of them having a single-processor configuration.

\subsubsection{Assimilation of the fractional cloud coverage $C$}

Results shown in Fig. 11 are the same as Fig. 8 but with the addition of the cloud coverage assimilation $C=99 \%$, $C=80 \%, C=50 \%$ and $C=20 \%$. Horizontal extensions $L_{x}$ and $L_{y}$ are set to $10 \mathrm{~km}$ and vertical resolution $\Delta z$ is set to $24 \mathrm{~m}$.
They show that 3DCLOUD is able to assimilate correctly fractional cloud coverage of stratocumulus for very different values of $C$, even though the extreme example with $C=20 \%$ is a fair weather cumulus field rather than a stratocumulus. For each value of $C$ assimilated, it is interesting to note that cloud base and cloud top heights are still localised around $600 \mathrm{~m}$ and $800 \mathrm{~m}$, respectively. Temperature vertical profiles are almost unchanged. The water mixing ratio vertical profiles decrease with the assimilated $C$ value.

\subsubsection{Effect of the outer scale $L_{\text {out }}$ and inhomogeneity parameter $\rho_{\tau}$ on the optical depth field}

We saw that 3DCLOUD can, at the end of step 1, simulate stratocumulus and cumulus fields with enough coherent statistics profiles. However, optical depth (for stratocumulus and cumulus) or IWC (for cirrus) generated during step 1 of 3DCLOUD does not show scale invariant properties observed in real cloud and often characterised by the spectral exponent $\beta_{1-\mathrm{D}}$ close to $-5 / 3$. As described in Sect. 2.2, it is the main task of the step 2 of 3DCLOUD, in addition to the adjustment of the mean and standard deviation of optical thickness or IWC. We focus on the DYCOMS2-RF01 case at the spatial resolution $\Delta x=50 \mathrm{~m}$ (Fig. 8c). The effective radius $R_{\text {eff }}$ is set to $10 \mu \mathrm{m}$ to compute optical depth from liquid water content. The mean optical depth of this initial field is set to 10 and we change the inhomogeneity parameter $\rho_{\tau}$ and the outer scale $L_{\text {out }}$ to 0.2 and $1 \mathrm{~km}$, respectively, for 


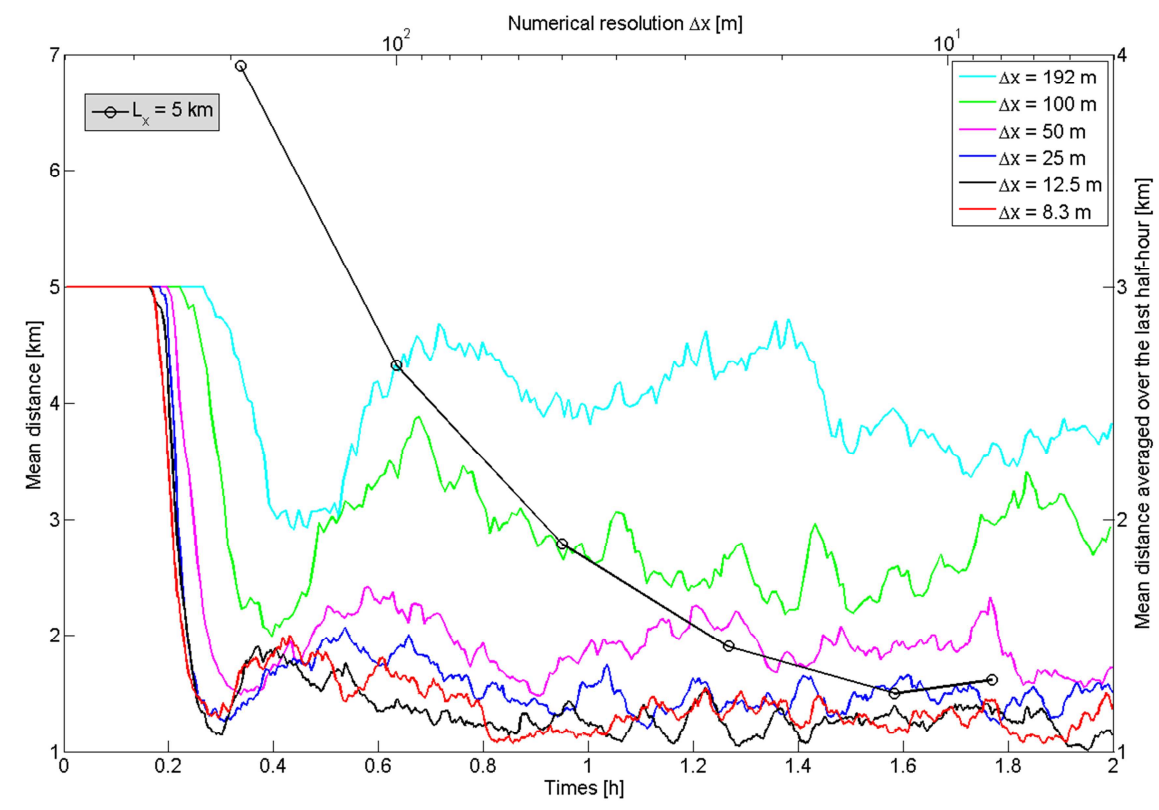

Figure 10. Time series of the mean distance between cloud areas for different horizontal numerical spatial resolutions (coloured lines) with a constant vertical numerical spatial resolution $(\Delta z=38.5 \mathrm{~m})$ and mean distance averaged over the last-half hour of a $2 \mathrm{~h}$ simulation as a function of numerical spatial resolution (black line with circles). The cumulus cloud is simulated by 3DCLOUD with assimilation of meteorological profiles based on the BOMEX case. Horizontal extensions are $L_{x}=L_{y}=5 \mathrm{~km}$ and vertical extension is $L_{z}=2700 \mathrm{~m}$.

case 1 to 0.7 and $1 \mathrm{~km}$ for case 2 and to 0.7 and $10 \mathrm{~km}$ for case 3. Figure 12 shows pseudo-albedo, mean power spectra, probability density function of optical depth fields, mean vertical profiles of the horizontally averaged optical depth for the three cases and a volume rendering of optical depth.

First, we notice that the pseudo-albedo of the initial optical depth field (see Fig. 12a) is smoother than the pseudoalbedo of case 1,2, and 3. Between cases 1 and 2, we clearly see an increase in heterogeneity as case 1 is a quasihomogenous stratocumulus with a small value of $\rho_{\tau}=0.2$ and case 2 is more inhomogeneous with a larger value of $\rho_{\tau}=0.7$. Between cases 2 and 3, we can see the effect of the outer scale. In accordance with smooth variations, the spectral slope of the initial optical depth is close to -3 for the $\left[10^{-3}: 10^{-2}\right] \mathrm{m}^{-1}$ wave number range (Fig. 12e). Cases 1, 2 and 3 present the proper spectral slope value of $-5 / 3$. For cases 1 and 2 , this slope is obtained for the $\left[10^{-3}: 10^{-2}\right] \mathrm{m}^{-1}$ wave number range, which is coherent with the imposed value of outer scale $L_{\text {out }}=1 \mathrm{~km}$. For case $3, L_{\text {out }}=10 \mathrm{~km}$, so the spectral slope should be $-5 / 3$ on the $\left[10^{-4}: 10^{-2}\right] \mathrm{m}^{-1}$ wave number range. However, we note that this spectral slope value is achieved only for the $\left[5 \times 10^{-3}: 10^{-2}\right] \mathrm{m}^{-1}$ wave number range, because we keep the phase angles unchanged in the 3DCLOUD algorithm.

In Fig. 12f, we represent the optical depth distributions. The initial optical depth distribution does not follow a common distribution, whereas the optical depth distribution for cases 1 and 2 are log-normal. Indeed, in the 3DCLOUD algorithm, a gamma distribution for the optical depth is imposed.
For case 2 and 3, optical depth distributions are very close, even if the outer scales are different. Thus, changing the $L_{\text {out }}$ value does not affect significantly the shape of optical depth distribution. In Fig. 12g, we can see that the horizontal mean optical depth profiles are quasi identical for all cases.

These results show undeniably the flexibility of the 3DCLOUD algorithm. Indeed, in step 2, 3DCLOUD is able, by mapping a theoretical gamma-distributed optical depth onto the optical depth field simulated at step1, to adjust, quasi-independently, the optical depth mean value, the inhomogenenity parameter value of optical depth and the spectral slope value of optical depth for $\left[1 / L_{\text {out }}: 1 / 2 \Delta x\right] \mathrm{m}^{-1}$ wave number range.

\subsection{Cirrus fields examples}

\subsubsection{Cirrus fields with assimilation of idealized meteorological profiles}

Including some modifications presented in Sect. 2.1.1, 3DCLOUD is also able to generate cirrus cloud. We briefly present in this section an example of ice water content (IWC) of cirrus with fallstreaks. For cirrus, we chose to generate IWC field instead of optical depth field as for stratocumulus or cumulus.

Figure 13 shows idealized vertical profiles of potential temperature, relative humidity, and horizontal velocity components assimilated by 3DCLOUD as well as the ice water path (IWP) simulated at step 1 by 3DCLOUD. It also shows 

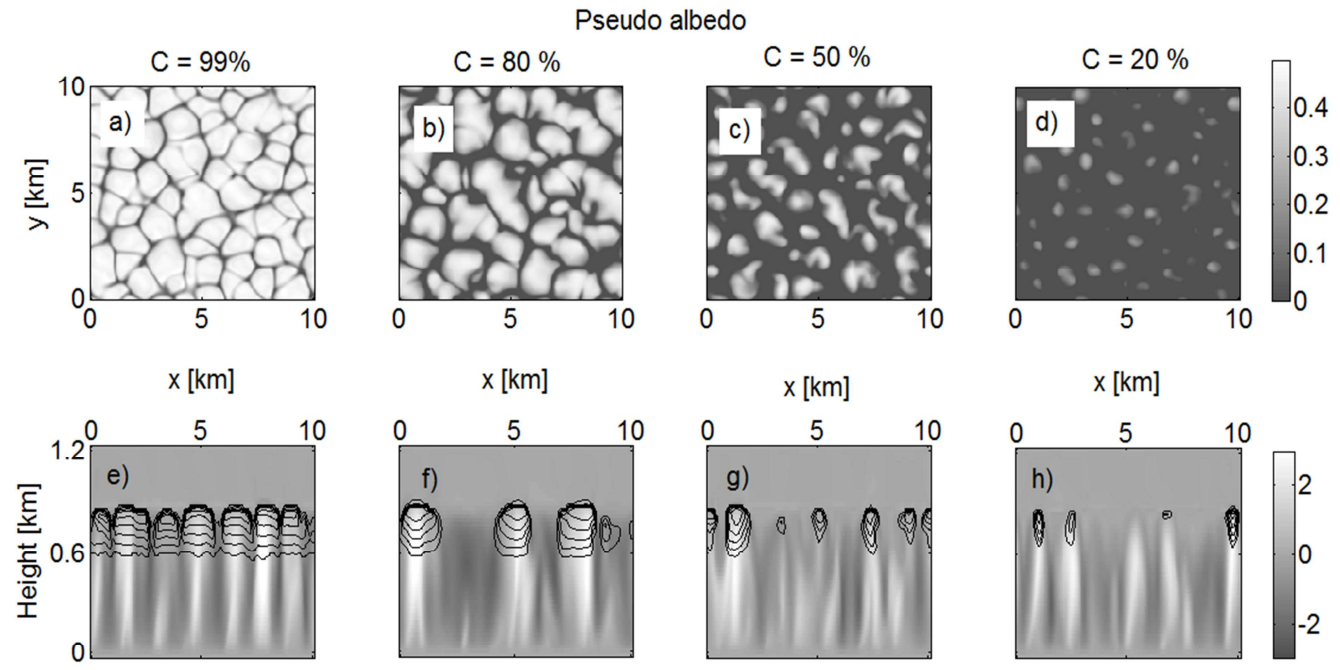

Vertical velocity $[\mathrm{m} / \mathrm{s}]$
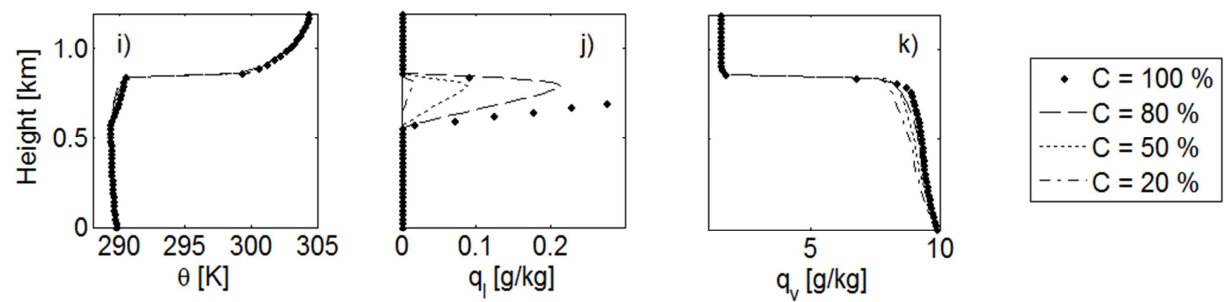

Figure 11. Same as Fig. $8 \mathrm{c}(\Delta x=50 \mathrm{~m})$, for different assimilated values of the cloud coverage: (a) and (e) $C=99 \%$, (b) and (f) $C=80 \%$, (c) and (G) $C=50 \%$ and (d) and (h) $C=20 \%$.

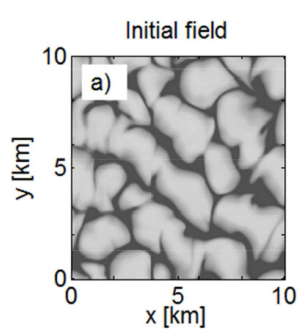

$$
\begin{gathered}
\rho_{\tau}=0.2 ; L_{\text {out }}=1 \mathrm{~km} \\
\quad \text { (case 1) }
\end{gathered}
$$

$$
\begin{gathered}
\rho_{\tau}=0.7 ; L_{\text {out }}=1 \mathrm{~km} \\
\quad(\text { case } 2)
\end{gathered}
$$
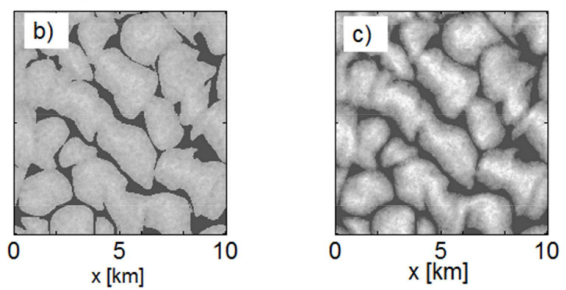

$$
\begin{aligned}
& \rho_{\tau}= 0.7 ; L_{\text {out }}=10 \mathrm{~km} \\
& \quad(\text { case } 3)
\end{aligned}
$$
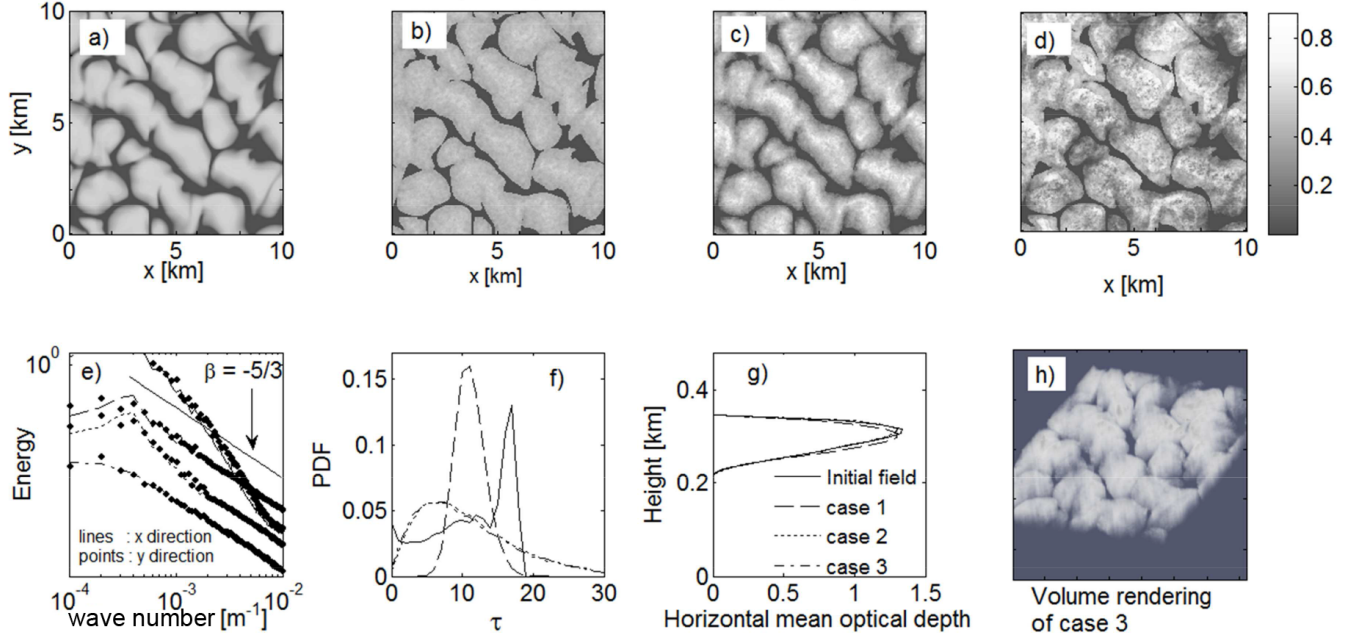

Figure 12. (a) Pseudo albedo estimated from optical depth (initial field) simulated in the step 1 by 3DCLOUD for the DYCOMS2-RF01 case (see Fig. 8c), (b), (c) and (d) pseudo albedo adjusted in the step 2 of 3DCLOUD for different values of the inhomogeneity parameter $\rho_{\tau}$ and of the outer scale $L_{\text {out }}$. (e) mean power spectra of optical depth along $x$ and $y$ directions. The power spectra are scaled for better visualization. (f) probability density function of optical depth, (g) mean vertical profiles of horizontally averaged optical depth and (h) volume rendering of optical depth for the case $3 . \rho_{\tau}$ and $L_{\text {out }}$ are 0.2 and $1 \mathrm{~km}$ for case $1,0.7$ and $1 \mathrm{~km}$ for case 2 and 0.7 and $10 \mathrm{~km}$ for case 3 , respectively. Solid lines, dotted lines, dashed lines and dash-dot lines indicate initial field, case 1, case 2 and case 3 fields, respectively. 

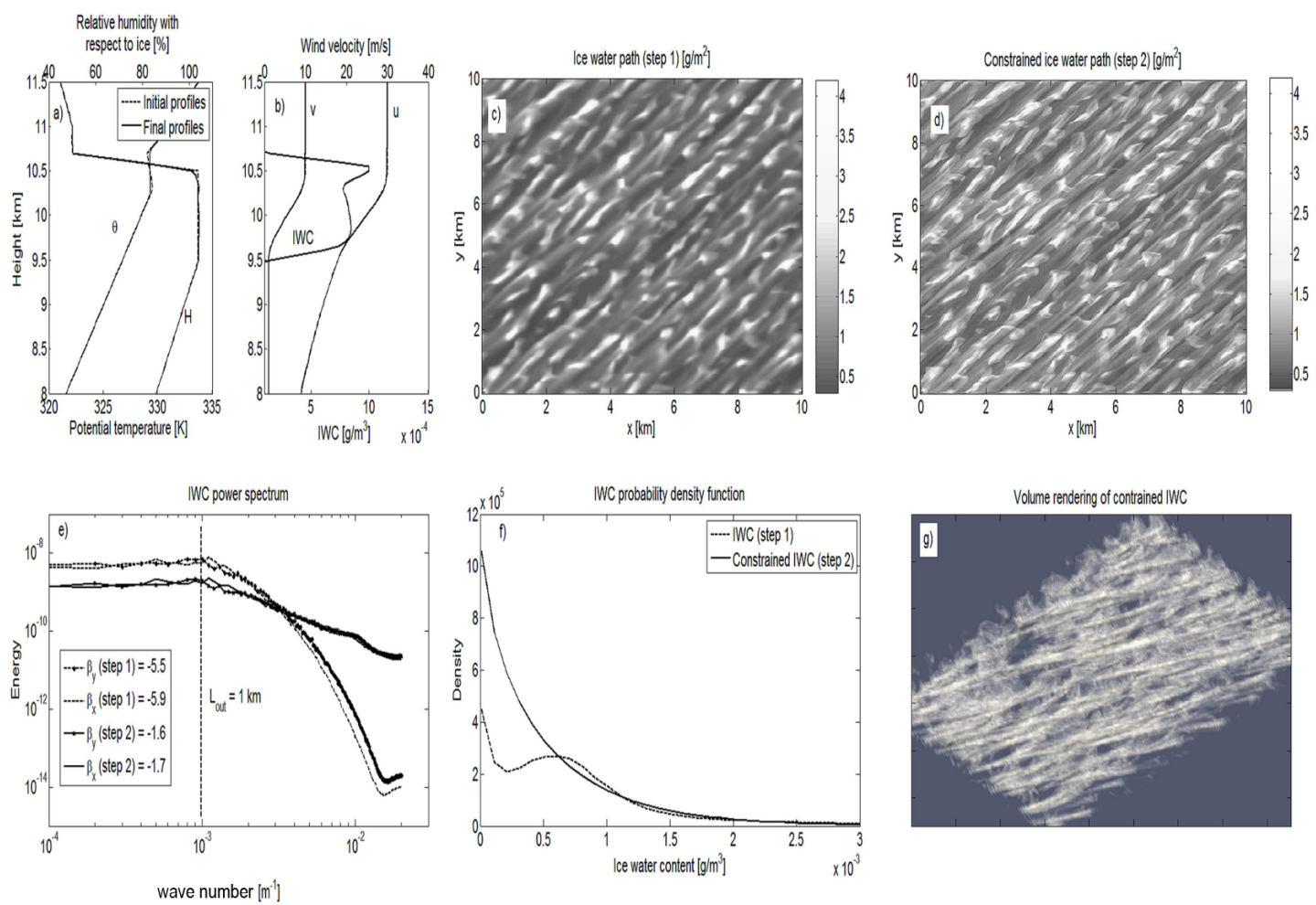

Figure 13. Idealized vertical profiles assimilated (dashed lines) and simulated (solid lines) by 3DCLOUD during step 1 of (a) the potential temperature and relative humidity and of (b) the horizontal velocity components and the ice water content (IWC), (c) ice water path (IWP) simulated by 3DCloud in step 1, (d) IWP simulated by 3DCloud in step 2, (e) mean power spectra of IWC along $x$ and $y$ directions after the step 1 and the step 2. (f) IWC probability density functions after step 1 and step 2. (g) IWC volume rendering after step 2. $\rho_{\text {IWC }}$ is set to 1 and $L_{\text {out }}$ is set to $1 \mathrm{~km}$. Number of iterations is 1000 .

the IWP simulated by 3DCLOUD during step 2, the initial and corrected mean power spectra, the initial and corrected probability density functions and the IWC volume rendering. Horizontal extensions $L_{x}=L_{y}$ and vertical extension $L_{z}$ are set to $10 \mathrm{~km}$ and $12.5 \mathrm{~km}$, respectively. Horizontal resolutions $\Delta x=\Delta y$ and vertical resolution $\Delta z$ are set to $24 \mathrm{~m}$ and $83.3 \mathrm{~m}$, respectively. $\overline{\mathrm{IWC}}$ is set to the value obtained at the end of step $1\left(0.54 \mathrm{mg} \mathrm{m}^{-3}\right)$. The inhomogeneity parameter $\rho_{\text {IWC }}$ is set to 1.0 and the outer scale $L_{\text {out }}$ to $1 \mathrm{~km}$.

Initial meteorological profiles assimilated by 3DCLOUD have been constructed in such a way that thin cirrus is generated between $9.5 \mathrm{~km}$ and $10.5 \mathrm{~km}$ with fallstreaks. Vertical profiles of potential temperature, and especially their vertical gradients under and above the cirrus are based on those proposed by Liu et al. (2003). In order to generate instabilities due to radiative cooling (not simulated by 3DCLOUD), we imposed a null vertical gradient of the potential temperature near the cirrus top height. We imposed a mean relative humidity with respect to ice (RHI) of $104 \%$ between $9.5 \mathrm{~km}$ and $10.5 \mathrm{~km}$. Just above the cloud, RHI is set to $50 \%$ then $20 \%$ near $12 \mathrm{~km}$ in altitude. Under the cirrus, RHI decreases with height to $85 \%$ near $8 \mathrm{~km}$. To generate fallstreaks, we imposed larger wind shear inside the cirrus than under the cirrus.
In Fig. 13, we note that IWP obtained after step 1 is smoother than IWP obtained after step 2, and that the initial IWC spectral slope value after step 1 is much smaller (around -5.5) than the corrected IWC spectral slope after step 2 (around -1.6) in the $\left[10^{-3}: 2 \times 10^{-2}\right] \mathrm{m}^{-1}$ wave number range. For wave number smaller than $1 / L_{\text {out }}=10^{-3} \mathrm{~m}^{-1}$, the power spectra are constant. The corrected IWC probability distribution is exponential-like distribution after step 2 . This is due to the larger value of $\rho_{\mathrm{IWC}}=1.0$ used in this example, compared to $\rho_{\tau}=0.7$ used for stratocumulus in Sect. 4.1.3.

\subsubsection{Cirrus field and wind shear}

We investigate briefly the aspect of cloud organization due to wind shear with 3DCLOUD model and with other stochastic models. We focus on the work of Marsham and Dobbie (2005) and of Hogan and Kew (2005). These two studies are very pertinent together. Indeed, based on RADAR retrievals of IWC from the Chilbolton $94 \mathrm{GHz}$ RADAR on 27 December 1999, which shows a strongly sheared ice cloud (named hereafter RC99 case), Marsham and Dobbie (2005) investigated shear effects by simulating the RC 99 case with the UK Met office LES. In contrast, Hogan and Kew (2005) 


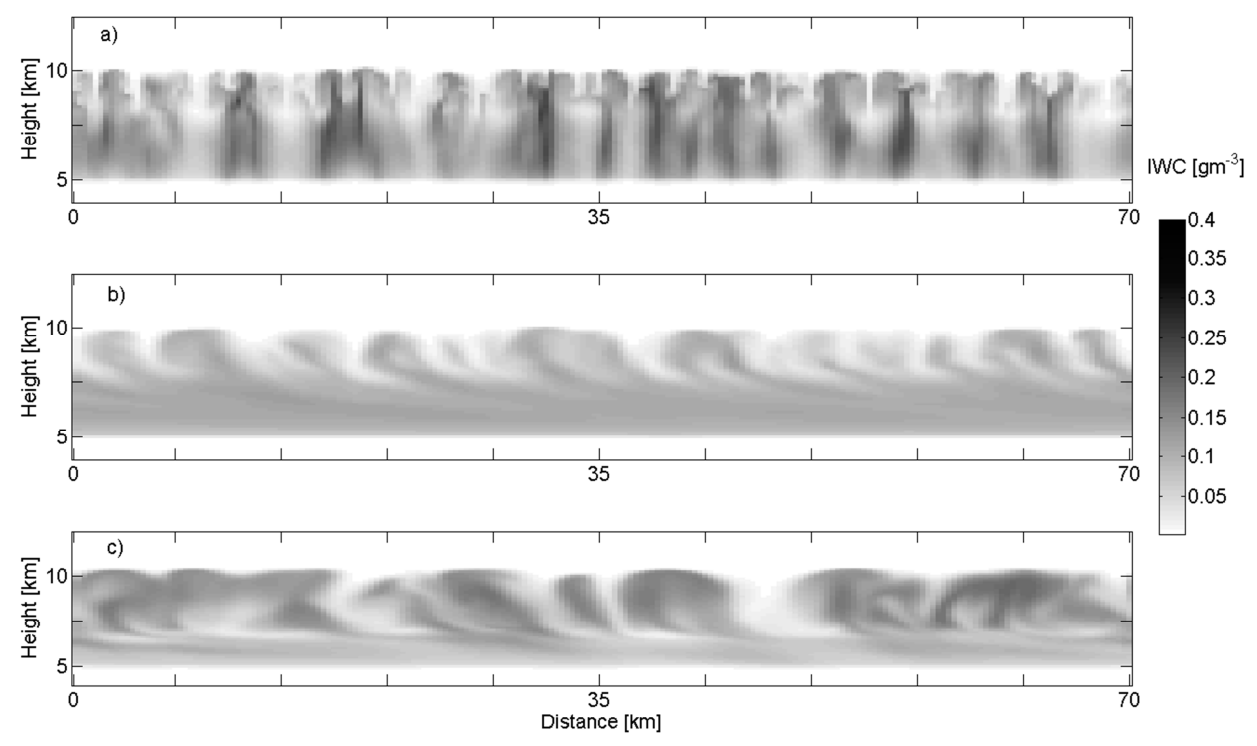

Figure 14. 2-D vertical slice of 3DCLOUD ice water content (IWC gm ${ }^{3}$ ) through a 3-D simulation at an angle parallel to the wind (a) RC99a case, (b) RC99 case and (c) RC99b case. Fields are obtained from simulations where $N_{x}=N_{y}=200$ and $N_{z}=66$ and $\Delta_{x}=\Delta_{y}=250 \mathrm{~m}$ and $\Delta_{z}=120 \mathrm{~m}$. Horizontal extensions are $L_{x}=L_{y}=50 \mathrm{~km}$ and vertical extension is $L_{z}=8 \mathrm{~km}$ between $4 \mathrm{~km}$ and $12 \mathrm{~km}$. Note that the 3DCLOUD fields are smooth because obtained at the first step of the algorithm.

used their Cloudgen model, a 3-D stochastic cloud model being able to simulate the structural properties of ice clouds. To configure 3DCLOUD in order to simulate the RC99 case, we assimilate meteorological profiles (potential temperature, horizontal wind velocity) based on those drawn in Fig. 2 in Marsham and Dobbie (2005). We run also the RC99a case with no wind (and therefore no wind shear), and the RC99b case where the potential temperature profile (drawn in Fig. 15 in Marsham and Dobbie, 2005) reduces atmospheric stability in order to give more extensive KelvinHelmholtz wave braking. All our simulations are done with $N_{x}=N_{y}=200$ and $N_{z}=66$ and $\Delta_{x}=\Delta_{y}=250 \mathrm{~m}$ and $\Delta_{z}=120 \mathrm{~m}$. Horizontal extensions are $L_{x}=L_{y}=50 \mathrm{~km}$ and vertical extension is $L_{z}=8 \mathrm{~km}$ between $4 \mathrm{~km}$ and $12 \mathrm{~km}$. Note that 3DCLOUD, Marsham and Dobbie (2005) and Hogan and Kew (2005) numerical resolution are $\Delta_{x}=\Delta_{y}=$ $250 \mathrm{~m}, \Delta_{x}=100 \mathrm{~m}$ and $\Delta_{x} \approx 780 \mathrm{~m}$, respectively. Note also that 3DCLOUD, Marsham and Dobbie (2005) and Hogan and Kew (2005) horizontal extensions are $L_{x}=L_{y}=50 \mathrm{~km}$, $L_{x}=50 \mathrm{~km}$ and $L_{x}=L_{y}=200 \mathrm{~km}$.

Figure 14 shows a 2-D vertical slice of 3DCLOUD IWC at an angle parallel to the wind of the RC99a case, the RC99 case and the RC99b case. Note that the 3DCLOUD fields are smooth because they are obtained at the first step of the algorithm. These three snapshots are very similar to those presented in Marsham and Dobbie (2005), allowing us to confirm that our basic atmospheric equations are correctly solved. In Fig. 14a, we can see small structures (few km) at above $7 \mathrm{~km}$, due to radiative cooling at the cloud top and latent heat release in the updraughts. Below, we can observe fallstreaks advected (or not if there is no wind) relative to their source in the convective layer by the shear. The shear homogenizes the fallstreaks. Figure $14 \mathrm{~b}$ clearly shows that 3DCLOUD simulations at the first step of the algorithm homogenize the fallstreaks a lot, certainly too much compared to the RADAR retrievals of IWC from the Chilbolton $94 \mathrm{GHz}$ RADAR on 27 December 1999 (see Fig. 1 in Hogan and Kew, 2005). Figure 14c shows the RC99b case where 3DCLOUD model is able to simulate Kelvin-Helmholtz wave breaking, a dynamic aspect difficult to simulate with purely stochastic models.

Figure $15 \mathrm{a}$ and $\mathrm{b}$ are the same as Fig. 14b but 3DCLOUD fields are obtained at the second step of the algorithm, with 1 -D spectral slope close to $-5 / 3$ from the outer scale $L_{\text {out }}=$ $15 \mathrm{~km}$ to the numerical scale $\Delta x=250 \mathrm{~m}$. In Fig. 15a, the mean value of 3-D IWC $\overline{\text { IWC }}=0.07 \mathrm{~g} \mathrm{~m}^{-3}$ and inhomogeneity parameter $\rho_{\mathrm{IWC}}=0.4$ for cloudy voxels are those computed from the 3-D IWC field obtained at step one of the 3DCLOUD algorithm. In Fig. 15b, inhomogeneity parameter $\rho_{\text {IWC }}$ is a function of height. Its values are roughly estimated from the Fig. 2c in Hogan and Kew (2005). Compared to Hogan and Kew (2005) simulations, 3DCLOUD snapshots show more details in the convective layer above $7 \mathrm{~km}$ as it simulates relatively well the convective cloud structures thanks to imposed numerical spatial resolution. By contrast to Fig. 15a, Hogan and Kew (2005) simulations show more details in the layer under $7 \mathrm{~km}$, where the shear-induce mixing is important. In order to obtain such details in the layer under $7 \mathrm{~km}$ with 3DCLOUD, we have to constrain it with ancillary data: those provided by the RADAR retrievals of IWC and shown on Fig. 2c in Hogan and Kew (2005). Indeed, if we force, in the second step of 3DCLOUD algorithm, the 


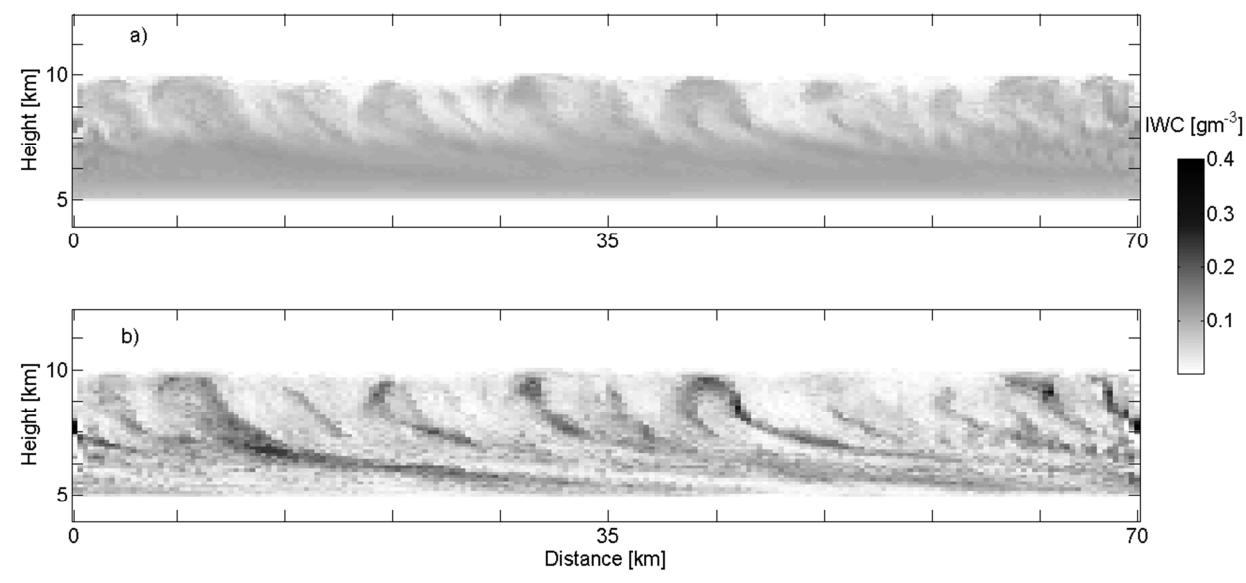

Figure 15. Same as Fig. 14b but after the second step of the algorithm, where 1-D spectral slope is close to $-5 / 3$ from outer scale $L_{\mathrm{out}}=$ $15 \mathrm{~km}$ to the numerical scale $\Delta x=250 \mathrm{~m}$. (a) mean of 3-D IWC is $\overline{\mathrm{IWC}}=0.07 \mathrm{gm}^{-3}$ and inhomogeneity parameter $\rho_{\mathrm{IWC}}=0.4$ for cloudy voxels. (b) same as (a) but with inhomogeneity parameter $\rho_{\mathrm{IWC}}$, function of height and derived from the 27 December 1999 RADAR measurements between 10:00 and 12:00 UTC crudely estimated from the Fig. 2c in Hogan and Kew (2005).
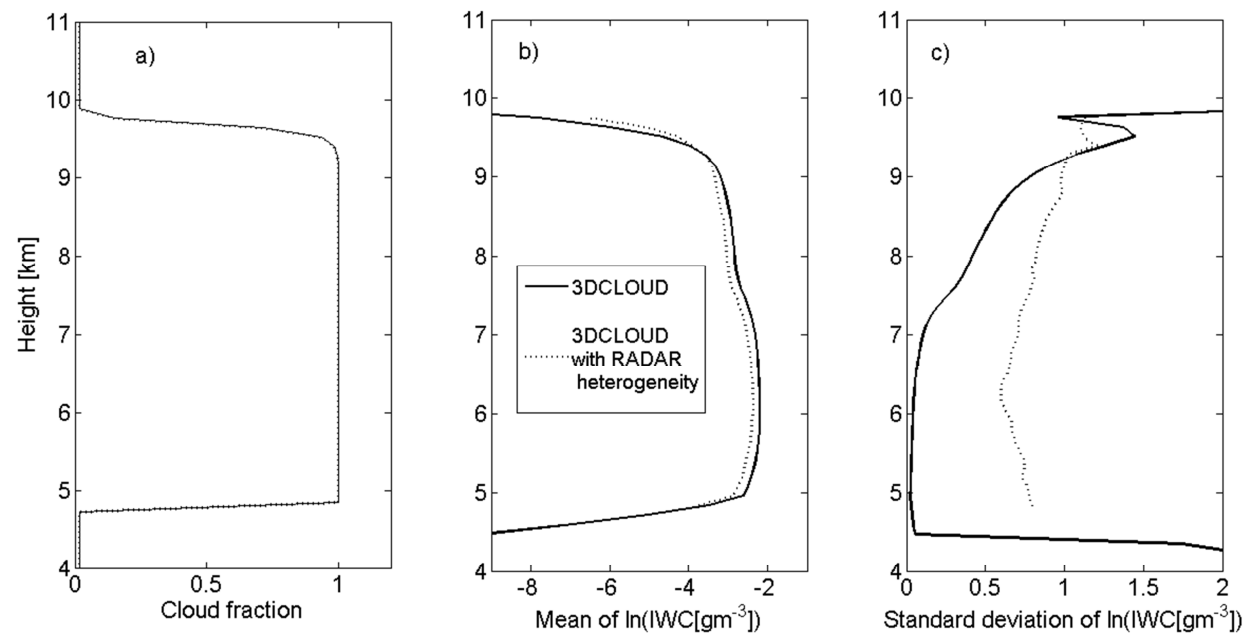

Figure 16. Vertical profiles for the RC99 case of (a) cloud fraction, (b) mean of $\ln (\mathrm{IWC})$ for the cloudy voxels and (c) standard deviation of $\ln$ (IWC) for cloudy voxels computed from two 3DCLOUD fields obtained at the second step of the algorithm. The solid lines indicate simulation where inhomogeneity parameter is $\rho_{\mathrm{IWC}}=0.4$ and the dotted lines indicate simulation where the parameter $\rho_{\mathrm{IWC}}$, function of height, is derived from the 27 December 1999 RADAR measurements between 10:00 and 12:00 UTC and crudely estimated from the Fig. 2c in Hogan and Kew (2005).

vertical inhomogeneity parameter to match the one estimated crudely from the RADAR retrievals, we obtain Fig. 15b. Details in the layer under $7 \mathrm{~km}$ of this snapshot are quite similar to those obtained by Hogan and Kew (2005).

Finally, Fig. 16, which is similar to Fig. 2 in Hogan and Kew (2005), shows vertical profiles of the cloud fraction, the mean of logarithm of IWC for the cloudy voxels and the standard deviation of logarithm of IWC for cloudy voxels computed from two 3DCLOUD fields obtained at the second step of the algorithm for RC99 case. As for Fig. 15, inhomogeneity parameter is $\rho_{\mathrm{IWC}}=0.4$ for the first cloud field and depends on height and it is derived from RADAR retrievals for the second case. For both cloud fields, the cloud coverage is equal to 1 between $5 \mathrm{~km}$ and $10 \mathrm{~km}$. RADAR retrievals of IWC shows that the cloud coverage is equal to 1 only between $5.5 \mathrm{~km}$ and $7 \mathrm{~km}$, and decreases to 0 at $10 \mathrm{~km}$. However, as discussed in Sect. 2.1.2, the current version of 3DCLOUD is not able to readily simulate fractional cloud coverage in the cirrus regime. For both cloud fields, vertical profiles of the mean IWC are quite similar and consistent with those retrieved from RADAR. The inhomogeneity parameter vertical profile simulated by the current version of 3DCLOUD is too small, leading to a smoothing of the layer 
under $7 \mathrm{~km}$ that can be improved if the vertical profile of the inhomogeneity parameter is known.

\section{Conclusions}

3DCLOUD is a flexible three-dimensional cloud generator developed to simulate with a personal computer and under Matlab environment, synthetic but realistic stratocumulus, cumulus and cirrus cloud fields. Simplified dynamic and thermodynamic laws allow the generation of realistic liquid or ice water content from meteorological profiles. The stochastic process with the Fourier framework allow us to provide ice water content or optical depth sharing similar statistical properties to those observed in real clouds such as the inhomogeneity parameter (set by the user) and the invariant scale properties characterised by a spectral slope close to $-5 / 3$ from the smaller scale (set by spatial resolution of grid computation) to the outer scale (set by the user). In order to simulate cloud structures, 3DCLOUD solves simplified basic atmospheric equations and assimilates the cloud coverage set by the user (only for the stratocumulus and cumulus regimes) and meteorological profiles (pressure, humidity, wind velocity) defined by the user.

The 3DCLOUD outputs were compared to LES ones for three classical test cases. We chose the case of DYCOMS2RF01, the case of BOMEX, and the case of ICMCP. For these cases, results show that 3DCLOUD outputs are relatively consistent with LES outputs, and confirm that the chosen basic atmospheric equations of 3DCLOUD are solved correctly. We also show that, under the condition that the user provides coherent meteorological profiles, 3DCLOUD algorithm is able to assimilate them and generate realistic cloud structures.

3DCLOUD is a very interesting research tool to better understand 3-D interactions between cloudy atmosphere and atmospheric radiation, which is of primary importance in order to make progress in the direct radiative problem (global climate models context) and in the inverse radiative problem (remote sensing context, development of the next generation of atmospheric sensors). For example, 3DCLOUD was used to quantify the impact of stratocumulus heterogeneities on polarized radiation measurements performed by POLDER/PARASOL (Cornet et al., 2013) as well as the influence of cirrus heterogeneities on brightness temperature measured by IIR/CALIPSO (Fauchez et al., 2013, 2014).

We still have to develop a stochastic process to generate 3$\mathrm{D}$ field of cloud effective radius. In a longer term, investigations will focus on the generation of 3-D mixed phase cloud and eventually on the simulation of 3-D rain rate. Another task will be to provide a FORTRAN code of 3DCLOUD, assumed to be faster than the current Matlab code.

\section{Code availability}

The source code of the 3DCLOUD algorithm is available online at http://wwwobs.univ-bpclermont.fr/atmos/fr/ restricted.

Please contact the authors for the password.

Acknowledgements. This work has been supported by the Programme National de Télédétection Spatiale (PNTS, http://www.insu.cnrs.fr/actions-sur-projets/ pnts-programme-national-de-teledetection-spatiale), grant no PNTS-2012-08 and by the Office National d'Etudes et de Recherches Aérospatiales (the French aerospace laboratory, ONERA). The authors thank Wolfram Wobrock and Valery Shcherbakov for the numerous fruitful discussions on physics and atmospheric dynamics. We also thank the two reviewers whose remarks largely improved the quality of this article.

Edited by: R. Neale

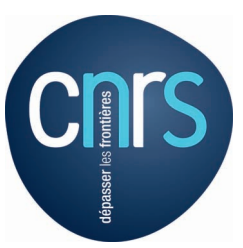

The publication of this article is financed by CNRS-INSU.

\section{References}

Alexandrov, M. D., Marshak, A., and Ackerman, A. S.: Cellular statistical models of broken cloud fields. Part I: Theory, J. Atmos. Sci., 67, 2125-2151, doi:10.1175/2010JAS3364.1, 2010.

Asai, T.: A numerical study of the air-mass transformation over the Japan Sea in winter, J. Met. Soc. Japan, 43, 1-15, 1965.

Barker, H. W. and Liu, D. M.: Inferring optical depth of broken clouds from Landsat data, J. Climate, 8, 2620-2630, 1995.

Barker, H. W., Wiellicki B. A., and Parker, L.: A parameterization for computing grid-averaged solar fluxes for inhomogeneous marine boundary layer clouds. Part II: validation using satellite data, J. Atmos. Sci., 53, 2304-2316, 1996.

Battaglia, A. and Mantovani, S.: Forward Monte Carlo computations of fully polarized microwave radiation in non isotropic media, J. Quant. Spectrosc. Rad. Transf., 95, 285-308, 2005.

Battaglia, A. and Tanelli, S.: DOMUS: DOppler MUltipleScattering Simulator, IEEE Trans. Geosci Rem. Sens., 49, 442450, 2011.

Benassi, A., Szczap F., Davis, A. B., Masbou, M., Cornet, C., and Bleuyard, P.: Thermal radiative fluxes through inhomogeneous cloud fields: A sensitivity study using a new stochastic cloud generator, Atmos. Res., 72, 291-315, 2004.

Bony, S. R., Colman, R., and Kattsov, V. M.: How well do we understand and evaluate climate change feedback processes?, J. Climate, 19, 3445-3482, 2006.

Bryan, G. H., Wyngaard, J. C., and Fritsch, J. M.: Resolution requirements for the simulation of deep moist convection, Mon. Weather Rev., 131, 2394-2416, 2003. 
Cahalan, R. F.: Bounded cascade clouds: albedo and effective thickness, Nonlin. Processes Geophys., 1, 156-167, doi:10.5194/npg1-156-1994, 1994.

Cahalan, R. F., Ridgway, W., Wiscombe, W. J., Bell, T. L., and Snider, J. B.: The albedo of fractal stratocumulus clouds, J. Atmos. Sci., 51, 2434-2455, 1994.

Carlin, B., Fu Q., Lohmann U., Mace G. G., Sassen K., and Comstock, J. M.: High cloud horizontal inhomogeneity and solar albedo bias, J. Climate, 15, 2321-2339, 2002.

Cheng, W. Y. Y., Wu, T., and Cotton, W. R.: Large-eddy simulations of the 26 November 1991 FIRE II cirrus case, J. Atmos. Sci., 58, 1017-1034, 2001.

Clothiaux, E. E., Barker, H. W., and Korolev, A. V.: Observing clouds and their optical properties, in: 3-D Radiative Transfer in Cloudy Atmospheres, edited by: Marshak, A. and Davis, A. B., Springer, 93-152, 2004

Collins, W. D. and Satoh, M.: Simulating global clouds, past, present, and future, in: Clouds in the Perturbed Climate System, edited by: Heintzenberg, J. and Charlson R. J., MIT press, 469486, 2009.

Cotton, W. R., Pielke, R. A., Walko, R. L., Liston, G. E., Tremback, C. J., Jiang, H., McAnelly, R. L., Harrington, J. Y., Nicholls, M. E., Carrio, G. G., and McFadden, J. P.: RAMS 2001: Current status and future directions, Meteorol. Atmos. Phys., 82, 5-29, 2001.

Cornet, C., Buriez, J.-C., Riedi, J., Isaka, H., and Guillemet, B.: Case study of inhomogeneous cloud parameter retrieval from MODIS data, Geophys. Res. Lett., 32, L13807, doi:10.1029/2005GL022791, 2005.

Cornet, C., Labonnote, L.-C., and Szczap, F.: Three-dimensional polarized Monte Carlo atmospheric radiative transfer model (3DMCPOL): 3-D effects on polarized visible reflectances of a cirrus cloud, J. Quant. Spectr. Radiative Transfer, 111, 174-186, doi:10.1016/j.jqsrt.2009.06.013, 2010.

Cornet, C., Szczap, F., Labonnote, L.-C., Fauchez, T., Parol, F., Thieuleux, F., Riedi, J., Dubuisson, P., and Ferlay, N.: Evaluation of cloud heterogeneity effects on total and polarized visible radiances as measured by POLDER/PARASOL and consequences for retrieved cloud properties, AIP Conf. Proc., 1531, 99-102, doi:10.1063/1.4804717, 2013.

Davis, A. B., Marshak, A., Cahalan, R. F., and Wiscombe, W. J.: Multifractal characterizations of non-stationarity and intermittency in geophysical fields, observed, retrieved or simulated, J. Geophys. Res., 54, 241-260, 1994.

Davis, A. B., Marshak, A., Wiscombe, W. J., and Cahalan, R. F.: Scale invariance of liquid water distribution in marine stratocumulus: Part I. Spectral properties and stationarity issues, J. Atmos. Sci., 53, 1538-1558, 1996.

Davis, A. B., Marshak, A., Cahalan, R. F., and Wiscombe, W. J.: The Landsat scale break in Stratocumulus as a three-dimensional radiative transfer effect: implication for cloud remote sensing, J. Atmos. Sci. 54, 241-259, 1997.

Davis, A. B., Marshak, A., Gerber, H., and Wiscombe, W. J.: Horizontal structure of marine boundary layer clouds from centimeter to kilometer scales. J. Geophys. Res., 104, 6123-6144, 1999.

Deardorff, J. W.: Stratocumulus-capped mixed layers derived from a three-dimensional model, Bound.-Lay. Meteorol., 18, 495-527, 1980.
Di Giuseppe, F. and Tompkins, A. M.: Effect of spatial organisation on solar radiative transfer in three dimensional idealised stratocumulus cloud fields, J. Atmos. Sci., 60, 1774-1794, 2003.

Emanuel, K. A.: Atmospheric Convection, Oxford university press, 1994.

Evans, K. F. and Wiscombe, W. J.: An algorithm for generating stochastic cloud fields from radar profile statistics, Atmos. Res. 72, 263-289, 2004.

Fauchez, T., Cornet, C., Szczap, F., and Dubuisson, P.: Assessment of cloud heterogeneities effects on brightness temperatures simulated with a 3-D Monte-Carlo code in the thermal infrared, AIP Conf. Proc. Vol. 1531, 75-78, doi:10.1063/1.4804711, 2013.

Fauchez, T., Cornet, C., Szczap, F., Dubuisson, P., and Rosambert, T.: Impact of cirrus clouds heterogeneities on top-of-atmosphere thermal infrared radiation, Atmos. Chem. Phys., 14, 5599-5615, doi:10.5194/acp-14-5599-2014, 2014.

Hill, P. G., Hogan R. J., Manners, J., and Petch, J. C.: Parametrising the horizontal inhomogeneity of ice water content using CloudSat observations, Q. J. R. Meteorol. Soc., 138, 1784-1793, 2012.

Hogan R. J. and Illingworth A. J. : Parameterizing ice cloud inhomogeneity and the overlap of inhomogeneities using cloud radar data, J. Atmos. Sci., 60, 756-767, 2003.

Hogan, R. J. and Kew, S. F.: A 3-D stochastic cloud model for investigating the radiative properties of inhomogeneous cirrus clouds, Q. J. R. Meteorol. Soc., 131, 2585-2608, 2005.

Holton, J. R.: An Introduction to Dynamic Meteorology, Fourth edition, Elsevier academic press, 2004.

Houze Jr., R. A.: Cloud Dynamics, Academic press, San Diego, 1993.

Illingworth, A. and Bony, S. R.: Observational strategies at mesoand large scales to reduce critical uncertainties in future cloud changes, in : Clouds in the Perturbed Climate System, edited by: Heintzenberg, J. and Charlson, R. J., MIT press, 511-530, 2009.

Kärcher, B. and Spichtinger, P.: Cloud-controling factors of cirrus, in: Clouds in the Perturbed Climate System, edited by: Heintzenberg, J. and Charlson R. J., MIT press, 235-268, 2009.

Lafont, D. and Guillemet, B.: Subpixel fractional cloud cover and inhomogeneity effects on microwave beam-filling error, Atmos. Res., 72, 149-168, doi:10.1016/j.atmosres.2004.03.013, 2004.

Liou, K. N.: An introduction to atmospheric radiation, Academic press, 2002.

Liu, H.-C., Wang, P. K., and Schlesinger, R. E.: A numerical study of cirrus clouds. Part II: effects of ambient temperature, stability, radiation, ice microphysics, and microdynamics on cirrus evolution, J. Atmos. Sci., 60, 1097-1119, 2003.

Marshak, A., Davis, A., Wiscombe, W. J., Ridgway, W., and Cahalan, R.: Biases in shortwave column absorption in the presence of fractal clouds, J. Climate, 11, 431-446, 1998.

Marsham J. H. and Dobbie, S.: The effects of wind shear on cirrus: a large-eddy model and radar case-study, Q. J. Roy. Meteor. Soc., 131, 2937-2955, 2005.

Mayer, B.: Radiative transfer in the cloudy atmosphere, European Physical Journal Conferences, 1, 75-99, 2009.

Meyers, M. P., Walko, R. L., Harrington, J. Y., and Cotton, W. R.: New RAMS cloud microphysics parameterization, Part II. The two-moment scheme, J. Atmos. Res., 45, 3-39, 1997.

Moeng, C. H.: A large-eddy-simulation model fort he study of planetary boundery-layer turbulence, J. Atmos. Sci., 41, 2052-2062, 1984. 
Oreopoulos, L. and Cahalan, R. F.: Cloud inhomogeneity from MODIS, J. Climate, 18, 5110-5124, 2005.

Penide, G., Giraud, V., Bouniol, D., Dubuisson, P., Duroure, C., Protat, A., and Cautenet, S.: Numerical simulation of the 7 to 9 September 2006 AMMA mesoscale convective system: Evaluation of the dynamics and cloud microphysics using synthetic observations. AMMA special issue, Q. J. Roy. Meteor. Soc., 136, 304-322, doi:10.1002/qj.558, 2010.

Pincus, R. and Evans, K. F.: Computational cost and accuracy in calculating three-dimensional radiative transfer: Results for new implementations of Monte Carlo and SHDOM, J. Atmos. Sci., 66, 3131-3146, 2009.

Pielke, R. A.: Mesoscale Meteorological Modeling, Academic press, 2002.

Pielke, R. A., Cotton, W. R., Walko, R. L., Tremback, C. J., Lyons, W. A., Grasso, L. D., Nieholls, M. E., Moran, M. D., Wesley, D. A., Lee, T. J., and Copeland, J. H.: A comprehensive meteorological modelling system-RAMS, Meteorol. Atmos. Phys., 49, 69-91, 1992.

Riddaway, R. W.: Numerical methods, available at: www.ecmwf.int/newsevents/training/rcourse_notes/ NUMERICAL_METHODS/NUMERICAL_METHODS/

Numerical_methods6.html (last access: 10 January 2014), 2001.

Sassen, K., Wang L., Starr, D. O. C., Comstock, J. M., and Quante, M.: A midlatitude cirrus cloud climatology from the facility for atmospheric remote sensing. Part V: Cloud structural properties, J. Atmos. Sci., 64, 2483-2501, 2007.

Siebesma, A. P., Bretherton, C. S., Brown, A., Chlond, A., Cuxart, J., Duynkerke, P. G., Jiang, H., Khairoutdinov, M., Lewellen, D., H.-Moeng, C., Sanchez, E., Stevens, B., and Stevens, D. E.: A Large-Eddy Simulation intercomparison study of shallow cumulus convection, J. Atmos. Sci., 60, 1201-1219, 2003.

Starr, D. O. C. and Cox, S. K.: Cirrus Clouds. Part I: A cirrus cloud model, J. Atmos. Sci., 42, 2663-2681, 1985.
Starr, D. O. C., Benedetti, A., Boehm, M., Brown, P. R. A., Gierens, K. M., Girard, E., Giraud, V., Jakob, C., Jensen, E., Khvorostyanov, V. I., Koehler, M., Lare, A., Lin, R.-F., Maruyama, K., Montero, M., Tao, W.-K., Wang, Y., and Wilson, D.: Comparison of cirrus cloud models: a project of the GEWEX Cloud System Study (GCSS) working group on cirrus cloud system. Proceedings of the 13th International Conference on Cloud and Precipitation (ICCP), Reno, NV, USA, 14-18 August 2000.

Stevens, B., H-Moeng, C., Ackerman A. S., Bretherton,C. S., Chlond, A., de Roode, S., Edwards, J., Golaz, J. C., Jiang, H., Khairoutdinov, M., Kirkpatrick, M. P., Lewellen, D. C., Lock, A L., Müller, F., Stevens, D. E., Whelan, E., and Zhu, P.: Evaluation of large-eddy simulations via observations of nocturnal marine stratocumulus, Mon. Weather Rev., 133, 1443-1462, 2005.

Szczap, F., Isaka, H., Saute, M., Guillemet, B., and Ioltukhovski, A.: Effective radiative properties of bounded cascade nonabsorbing clouds: Definition of the equivalent homogeneous cloud approximation, J. Geophys. Res., 105, 20617-20634, doi:10.1029/2000JD900146, 2000.

Várnai, T. and Marshak, A.: Observations of three-dimensional radiative effects that influence MODIS cloud optical thickness retrievals, J. Atmos. Sci., 59, 1607-1618, 2002.

Várnai, T. and Marshak, A.: View angle dependence of cloud optical thickness retrieved by MODIS, J. Geophys. Res., 112, D06203, doi:10.1029/2005JD006912, 2007.

Venema, V., Meyer, S., García, S. G., Kniffka, A., Simmer, C., Crewell, S., Löhnert, U., Trautmann, T., and Macke A.: Surrogate cloud fields generated with the Iterative Amplitude Adapted Fourier Transform algorithm, Tellus A, 58A, 104-120, 2006.

Yamaguchi, T. and Feingold, G.: Technical note: Large-eddy simulation of cloudy boundary layer with the Advanced Research WRF model, J. Adv. Model. Earth Syst., 4, M09003, doi:10.1029/2012MS000164, 2012.

Yang, H., Dobbie, S., Mace, G. G., Ross, A., and Quante, M.: GEWEX Cloud System Study (GCSS) cirrus cloud working group: development of an observation-based case study for model evaluation, Geosci. Model Dev., 5, 829-843, doi:10.5194/gmd-5-829-2012, 2012. 